\title{
'Candidatus Liberibacter asiaticus' and Its Vector, Diaphorina citri, Augment the Tricarboxylic Acid Cycle of Their Host via the $\gamma$-Aminobutyric Acid Shunt and Polyamines Pathway
}

\author{
Yasser Nehela, ${ }^{1,2}$ and Nabil Killiny ${ }^{1,+}$ \\ ${ }^{1}$ Department of Plant Pathology, Citrus Research and Education Center, University of Florida, 700 Experiment Station Rd., Lake \\ Alfred, FL 33850, U.S.A. ${ }^{2}$ Department of Agricultural Botany, Faculty of Agriculture, Tanta University, Tanta, Egypt
}

Accepted 28 September 2018.

\begin{abstract}
Huanglongbing (HLB), a destructive citrus disease, is associated with 'Candidatus Liberibacter asiaticus', which is transmitted by the Asian citrus psyllid Diaphorina citri. Both ' $\mathrm{C} a$. L. asiaticus' and its vector manipulate the host metabolism for their benefit, to meet their nutritional needs and neutralize the host defense responses. We used a targeted gas chromatography-mass spectrometry-based method to explore the connection between the tricarboxylic acid (TCA) cycle, $\gamma$-aminobutyric acid (GABA) shunt, and polyamines (PAs) pathways in citrus. ' $\mathrm{Ca}$. L. asiaticus' and $\mathrm{D}$. citri accelerated the conversion of $\alpha$-ketoglutarate to glutamate, then to GABA, causing an accumulation of GABA in the cytosol. In silico analysis showed that the citrus genome possesses a putative GABA permease that connects the GABA shunt with the TCA cycle and supports the accumulation of succinate, fumarate, and citrate. Additionally, the PAs biosynthetic pathway might be connected directly to the TCA cycle, through the production of fumarate, or indirectly, via enhancement of GABA shunt. Taken together, we suggest that GABA shunt and PAs pathways are alternative pathways that contribute to the flux toward succinate rather than an intact TCA cycle in citrus. Both ' $\mathrm{Ca}$. L. asiaticus' and its vector enhance these pathways. This study provides more insights into citrus responses to the HLB pathosystem and could be a further step toward clues for understanding the nutritional needs of ' $\mathrm{Ca}$. L. asiaticus', which could help in culturing ' $C a$. L. asiaticus'.
\end{abstract}

Vector-borne phytopathogens cause many destructive diseases in economic crops worldwide. For instance, Huanglongbing (HLB, also known as citrus greening) is the most serious bacterial disease in citrus across many different geographical regions worldwide (Gottwald 2010). HLB is costing growers over $\$ 4$ billion annually, and thousands of citrus industry workers have lost their jobs (Gottwald 2010).

${ }^{\dagger}$ Corresponding author: N. Killiny; E-mail: nabilkilliny@ufl.edu

Funding: This work was generously supported by the United States Department of Agriculture National Institute of Food and Agriculture (201670016-24844). Y. Nehela was supported with a scholarship from the Egyptian government.

*The $\boldsymbol{e}$-Xtra logo stands for "electronic extra" and indicates that four supplementary figures and four supplementary tables are published online.

@ 2019 The American Phytopathological Society
Although Koch's postulates have not been fulfilled due to difficulty in culturing the putative bacterium, circumstantial evidence indicates that HLB is associated with a plantfastidious, phloem-limited, pathogenic bacterium given provisional Candidatus status, 'Candidatus Liberobacter spp.', a member of gram-negative $\alpha$-proteobacteria, and later changed to 'Candidatus Liberibacter spp.' (Bové 2006; Garnier et al. 2000; Gottwald 2010; Jagoueix et al. 1994). Taxonomically, based on the geographical distribution and the characteristic $16 \mathrm{~S}$ rDNA sequence, three of 'Candidatus Liberibacter' species have been proposed to be associated with HLB: ' $\mathrm{Ca}$. L. asiaticus' in Asia and the Americas (Bové 2006; Gottwald 2010), ' $C a$. L. africanus' in Africa (da Graça 1991), and ' $C a$. L. americanus' in Brazil (do Carmo Teixeira et al. 2005). Among the three species, ' $\mathrm{Ca}$. L. asiaticus' is the most dominant species, causing huge economic losses to citrus production worldwide (Bové 2006; Gottwald 2010). HLB affects most, if not all, citrus varieties; however, certain varieties have been reported to be more susceptible than others (Cevallos-Cevallos et al. 2012; Folimonova et al. 2009; Hijaz et al. 2016; Killiny 2017; Killiny and Hijaz 2016; Killiny et al. 2017b, 2018a). To our knowledge, the mechanisms or the internal factors responsible for HLB susceptibility or tolerance in citrus are yet to be explored.

Although ' $C a$. Liberibacter spp.' are mainly transmitted by psyllids, they can be transmitted by graft inoculation, but there is no evidence for transmission by seeds (Halbert and Manjunath 2004). Two psyllid vectors are responsible for the spread of HLB (do Carmo Teixeira et al. 2005): the Asian citrus psyllid Diaphorina citri Kuwayama (Hemiptera: Liviidae) in Asia and the Americas (Bové 2006; Gottwald 2010) and the African psyllid Trioza erytreae Del Guercio (Hemiptera: Triozidae) in Africa (da Graça 1991).

Previously, we described the chemical composition of citrus phloem sap because it contains all the required nutrients for multiplication of ' $C a$. L. asiaticus' and its vector, D. citri (Hijaz and Killiny 2014). Citrus phloem sap contains many sugars (sucrose, glucose, and inositol), organic acids (succinic, malic, benzoic, and citric acids), and about 20 proteinogenic amino acids (PAAs) (Hijaz and Killiny 2014). It has been suggested that the role of plant metabolites in plant-pathogen-vector interactions is to secure the energy requirements for plant defense responses (Bolton 2009; Kangasjärvi et al. 2012); however, this is not the main role. The changes in plant metabolites might contribute directly to plant defense responses. Our previous studies showed that both ' $C a$. L. asiaticus' infection and $D$. citri 
infestation can cause many biochemical and metabolic alterations in citrus plants. For example, both ' $\mathrm{Ca}$. L. asiaticus' infection and $D$. citri infestation altered the volatile organic compounds (Hijaz et al. 2013), amino acids, organic acids, fatty acids (Killiny and Nehela 2017a), leaf pigments (Killiny and Nehela 2017b), and phytohormones (Nehela et al. 2018). However, it remains unclear whether these changes benefit ' $\mathrm{Ca}$. L. asiaticus' and its vector or if they are a plant response, or both. Nonetheless, in the HLB-infected citrus plants, the existing defense mechanisms, however advanced, are usually not enough to overcome the harmful effects of ' $C a$. $\mathrm{L}$. asiaticus'.

In citrus, the vital and complex roles of leaf metabolites involved in plant defense response against various biotic stressors have been reported. These metabolites included amino acids (Cevallos-Cevallos et al. 2011, 2012; Killiny and Hijaz 2016; Killiny and Nehela 2017a; Malik et al. 2014; Slisz et al. 2012), polyamines (PAs) (Malik et al. 2014), organic acids (Cevallos-Cevallos et al. 2011; Killiny and Nehela 2017a; Slisz et al. 2012), fatty acids (Cevallos-Cevallos et al. 2012; Killiny and Nehela 2017a), phytohormones (Nehela et al. 2018; Rosales and Burns 2011), and other secondary metabolites. However, the contribution of nonproteinogenic amino acids (NPAAs) and PAs pathways in citrus defense responses are poorly understood.

Biochemically, the NPAAs are those not encoded or found in the genetic code of an organism and are not normally found as products of protein hydrolysis (Bell 2003). In eukaryotes, in addition to the 21 common amino acids used for protein biosynthesis, plants also produce numerous NPAAs. Over 140 amino acids are known to occur naturally in plants, and thousands more may occur in nature or be synthesized in the laboratory. Many NPAAs are important because they are highly recognizable as intermediates or end products of primary metabolism in both plants and animals (Bell 2003). In addition, NPAAs form posttranslationally in proteins and play many physiological roles in plants. For example, the NPAA $\gamma$-aminobutyric (GABA) was detected in the phloem sap of citrus (Hijaz and Killiny 2014). Additionally, many other NPAAs, including octopamine, synephrine, tyramine, $\mathrm{N}$-methyltyramine, hordenine, and putrescine were found in citrus leaves (Wheaton and Stewart 1970), and they could play a key role in citrus defense responses to HLB (Killiny and Nehela 2017a).

Additionally, PAs are ubiquitous, aliphatic amines, polycationic, and low-molecular weight, nitrogen-containing compounds found in all living organisms (Cohen 1998). For instance, putrescine (di-amine), spermidine (triamine), and spermine (tetra-amine) are the major PAs and occur ubiquitously in plants (Kaur-Sawhney et al. 2003). At physiological $\mathrm{pH}$, PAs are positively charged; therefore, they are known to bind to negatively charged molecules, such as nucleic acids, acidic phospholipids, and various types of proteins (Cohen 1998). PAs are implicated in several biological processes for growth and development in both prokaryotes and eukaryotes (Kaur-Sawhney et al. 2003; Tiburcio et al. 1997). Moreover, PAs modulate one or more plant responses to various environmental (Kaur-Sawhney et al. 2003) and biotic stressors, including plant-microbe interactions. It has been reported that the PAs pathway is altered after phytopathogen infections (Walters 2003a and b).

Although there are many studies focused on the metabolic effects of ' $\mathrm{Ca}$. L. asiaticus' infection on its vector, the effect of ' $C a$. L. asiaticus' infection on energy metabolism and the tricarboxylic acid (TCA) cycle metabolic pathway of its host plant is poorly studied. For instance, it has been recently shown that citrate and other TCA cycle intermediates are the main energy sources for Liberibacter crescens growth on chemically defined media (Cruz-Munoz et al. 2018). Furthermore, our previous studies showed that ' $\mathrm{Ca}$. L. asiaticus' infection exploits the energy metabolism, defense responses, and TCA cycle (also known as the Krebs cycle or citric acid cycle) of its insect vector, D. citri (Killiny et al. 2017a, 2018b; Lu and Killiny 2017). However, more studies are required to explore the roles of the TCA cycle metabolic pathway in plant defenses and in fulfilling the nutritional needs of $D$. citri and ' $C a$. L. asiaticus'.

Herein, we describe a comprehensive study on the effect of ' $C a$. L. asiaticus' infection on the TCA cycle, NPAAs, and PAs of its host plant, including the different natural scenarios (healthy, 'Ca. L. asiaticus'-infected, D. citri-infested, and double-attacked plants). We believe that the changes in different levels of leaf metabolites, particularly those involved in the TCA cycle, could also affect the abundance of both NPAAs and PAs and vice versa. Our hypothesis is that both ' $\mathrm{Ca}$. L. asiaticus' infection and $D$. citri infestation induce metabolic changes in the TCA-associated compounds, NPAAs, and PAs in citrus plants but these changes could be different under different stresses. In addition, we hypothesize that both the GABA shunt and the TCA cycle are functionally linked and the alteration in some metabolite levels, particularly the NPAAs and PAs, could lead indirectly to greater changes in the TCA cycle metabolic pathway. This study is a further step toward clues for understanding the nutritional needs of ' $\mathrm{Ca}$. L. asiaticus', which could help in culturing ' $\mathrm{Ca}$. L. asiaticus'. In addition, knowledge of the relationships between these pathways in the HLB pathosystem may clarify the defense mechanisms of citrus against ' $C a$. L. asiaticus' and $D$. citri in order to find novel, sustainable strategies for HLB management.

\section{RESULTS}

NPAAs and PAs, PAAs, and TCA-associated compounds were detected in citrus leaves.

We used a targeted gas chromatography-mass spectrometry (GC-MS)-based method to study the effect of one or both ' $\mathrm{Ca}$. L. asiaticus' infection and D. citri infestation on the NPAAs, PAs, TCA-associated compounds, and some PAAs implicated in the GABA shunt cycle and PAs pathway. We focused on these compounds after derivatization with methyl chloroformate (MCF). MCF derivatization resulted in highly reproducible chromatograms (Fig. 1A). Overall, we detected 19 different compounds in all studied treatments (Fig. 1A). Although more than 12 authentic reference standards of NPAAs and PAs were successfully derivatized with MCF and were detected using GC-MS, only nine of them were detected in citrus leaves, whereas $\alpha$-aminobutyric acid, $\beta$-alanine, and $N$ acetyl cysteine were not detected or were below the limit of detection. In addition, we detected four organic acids implicated in the TCA cycle (citric acid, 2-ketoglutaric acid, succinic acid, and fumaric acid) and six PAAs involved in the GABA shunt and other PAs pathways. These detected PAAs included L-proline, L-serine, L-glutamine, L-glutamic acid, L-cysteine, and L-tyrosine. Furthermore, L-arginine was detected only in ' $C a$. L. asiaticus'-infected plants (data not shown) after the derivatization with trimethylsilylation (TMS), whereas it was not detected at all when derivatized with MCF. The retention times, linear retention indices, and the main fragments of all detected compounds are shown in Supplementary Table S1.

\section{' $C a$. L. asiaticus' infection induces greater changes the total content of NPAAs and PAs, PAAs, and TCA-associated compounds in citrus leaves.}

The total targeted PAAs content was significantly increased in all treatments compared with control plants; however, ' $\mathrm{Ca}$. L. 
asiaticus'-infected trees had the highest PAA levels, followed by $D$. citri-infested and double-attacked plants $(P<0.0001)$ (Fig. 1B). Likewise, the total detected TCA-associated compounds were significantly higher in all treatments compared with control plants. Nevertheless, the induction of these compounds was significantly greater in the presence of ' $\mathrm{Ca}$. L. asiaticus' (' $\mathrm{Ca}$. L. asiaticus'-infected and double-attacked plants $)$, followed by $D$. citri-infested plants $(P<0.0001)$ (Fig. 1C). On the other hand, infestation alone did not influence the total NPAA and PA content, but the presence of ' $\mathrm{Ca}$. L. asiaticus' induced accumulation of these compounds in both 'Ca. L. asiaticus'-infected and double-attacked plants $(P<$ 0.0001) (Fig. 1D).

' $C a$. L. asiaticus' infection alters the NPAAs and PAs in citrus leaves.

After MCF derivatization, nine NPAA and PA compounds were detected among the four studied treatments (Fig. 2). The nonstandardized two-way hierarchical cluster analysis (HCA) showed that the peak areas of detected compounds ranged from $4.0 \times 10^{4}$ to $11.0 \times 10^{8}$ (Fig. $2 \mathrm{~A}$ ). Synephrine was the most abundant metabolite among all NPAAs and PAs in all studied treatments and was separately clustered in the top of the cluster dendrogram (Fig. 2A). On the other hand, seven metabolites (of nine compounds) had a small peak area less than $1.4 \times 10^{7}$. Ornithine from $D$. citri plants had the lowest peak area (approximately $4.0 \times 10^{4}$ ) among all detected metabolites (Fig. 2A).

Additionally, the standardized two-way HCA combined with analysis of variance (ANOVA) and Tukey's honestly significant difference (HSD) test (using the means of peak areas of individual NPAAs and PAs) were used to differentiate the individual metabolites among the studied treatments (Fig. 2B). The differences in the metabolite abundances were also visualized and were presented as a heat map. The total HCA dendrogram among treatments (Fig. 2B, bottom) showed that the metabolite profile of citrus leaves from ' $\mathrm{Ca}$. L. asiaticus'-infected plants was closer to the profile of double-attacked ones (more than

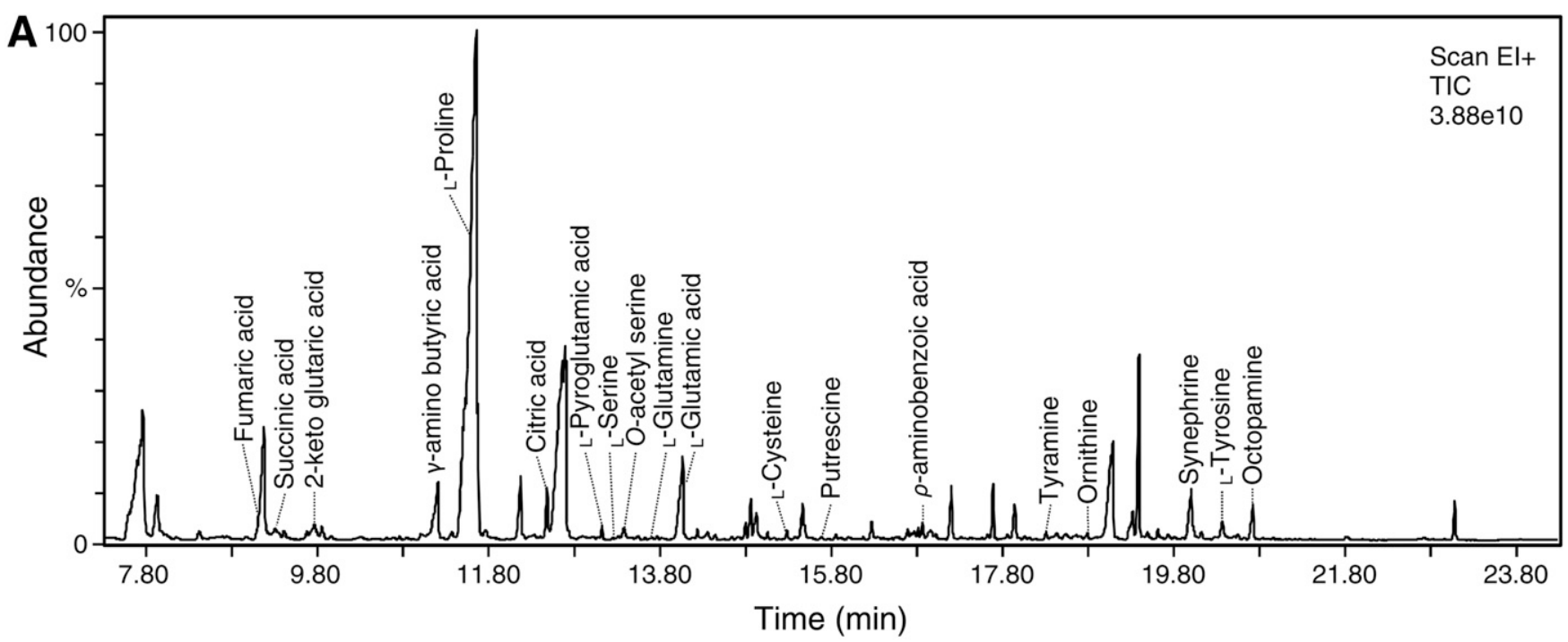

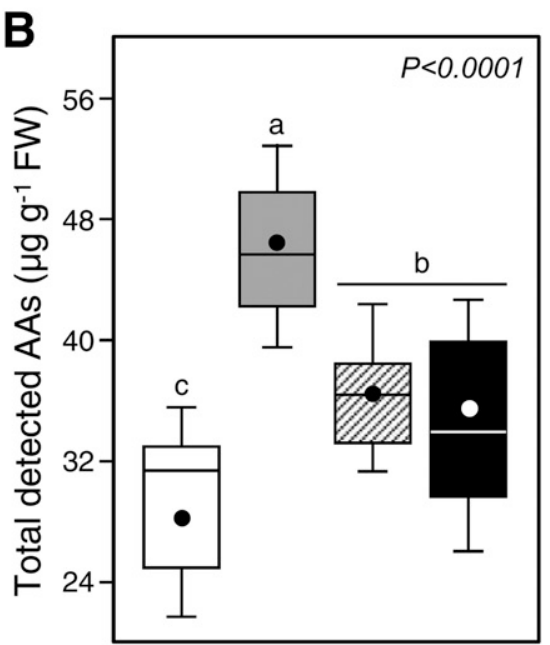

Total detected PAAs

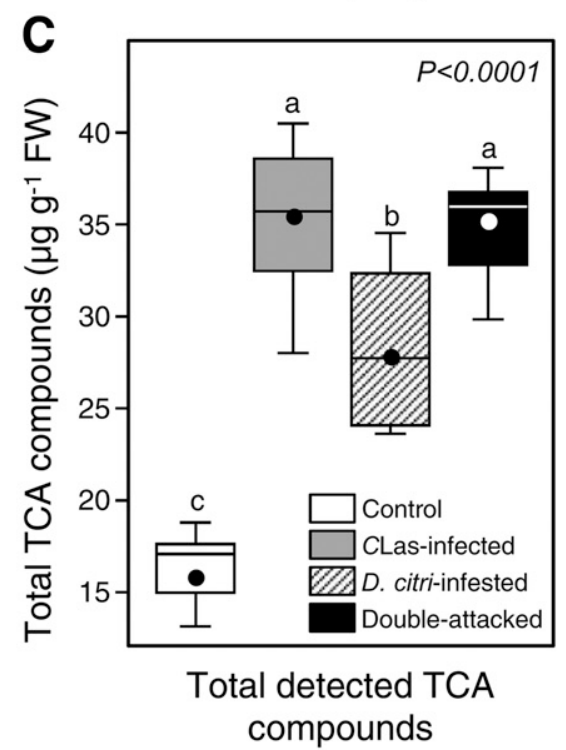

Fig. 1. Detected proteinogenic amino acids (PAAs), nonproteinogenic amino acids (NPAAs), polyamines (PAs), and tricarboxylic acid (TCA)-associated compounds of Valencia sweet orange (Citrus sinensis) leaves after derivatization with methyl chloroformate. A, Representative chromatogram of 19 detected metabolites in healthy citrus leaves, using gas chromatography-mass spectrometry (GC-MS), running in full-scan mode. B, Concentrations of total PAAs, C, total TCA-associated compounds, and D, total NPAA and PAs groups detected in Valencia sweet orange $(C$. sinensis) after either infection with ' $C a n d i d a t u s$ Liberibacter asiaticus' (CLas), infestation with Diaphorina citri, or both, using GC-MS $(n=10)$. Horizontal thick lines indicate the medians, black or white dots indicate the means, boxes show the interquartile ranges including 25 to $75 \%$ of the values, whiskers reflect the highest and the lowest value of data. Different letters indicate statistically significant differences among treatments, using the Tukey-Kramer honestly significant difference test $(P<0.05)$. 

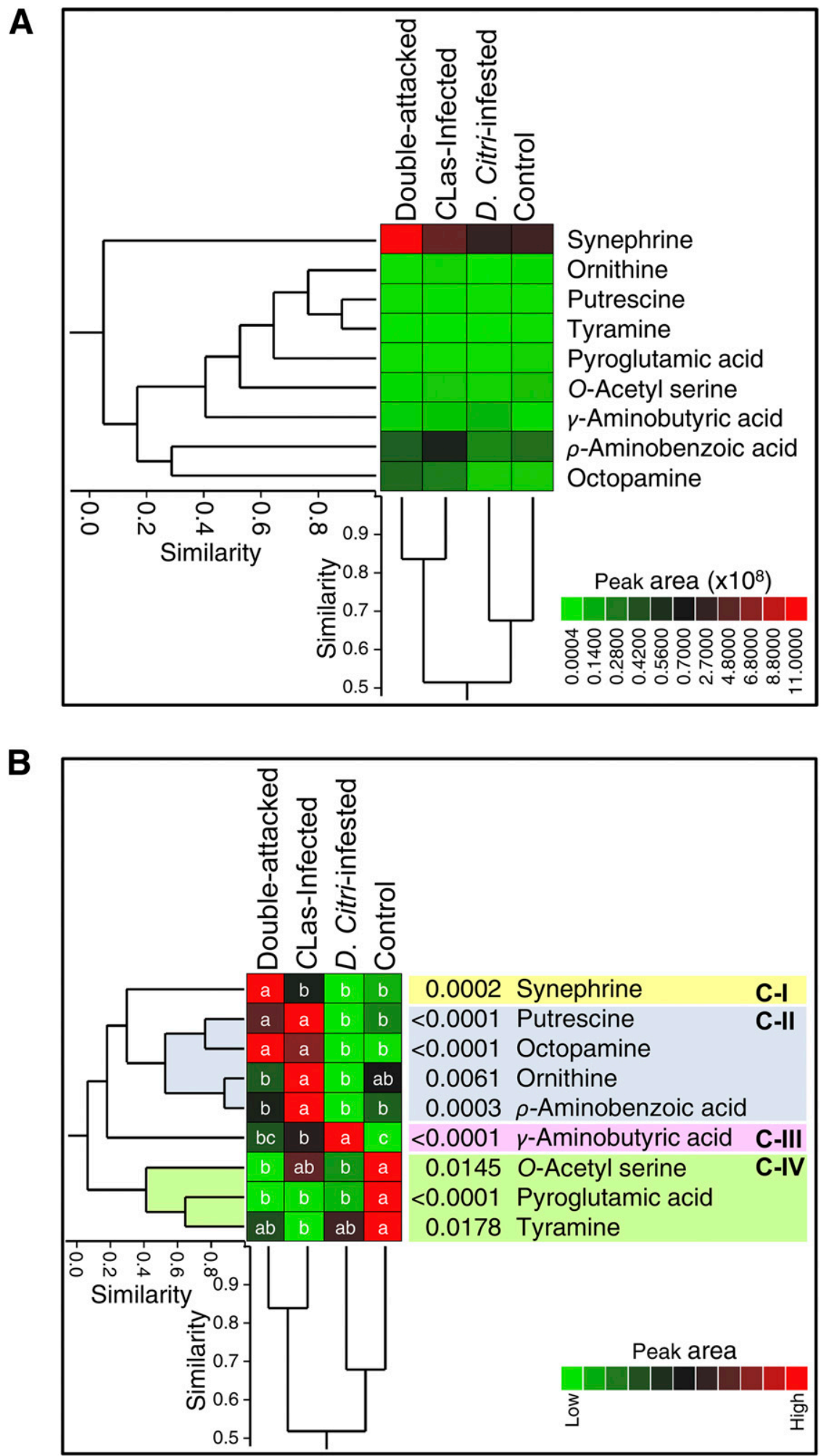

Fig. 2. Two-way hierarchical cluster analysis (HCA) of individual nonproteinogenic amino acids and polyamines detected in Valencia sweet orange (Citrus sinensis) leaf extracts after either infection with 'Candidatus Liberibacter asiaticus' (CLas), infestation with Diaphorina citri, or both, using gas chromatography-mass spectrometry $(n=10)$. A, HCA using the nonstandardized means of individual metabolites and B, HCA using the standardized means of individual metabolites of the matrices for the four studied treatments. The detected compounds separated into four clusters, designated cluster I (C-I) to cluster IV (C-IV). The differences in the metabolite abundances between the four treatments are visualized in the heat-map diagram. Rows represent the individual metabolites, while columns represent the treatments. Metabolites and treatments were organized using HCA based on similarities in auto-scaled values and correlations, respectively, with $95 \%$ confidence between groups from the discriminant function analysis, to construct the similarity dendrograms. In B, different letters indicate statistically significant differences among treatments, while cells with the same letter signify no significant differences among them, using the Tukey-Kramer honestly significant difference test $(P<0.05)$. The $P$ values are listed to the right side of the heat map. 
$80 \%$ similarity), whereas that of $D$. citri-infested plants was closer to control (less than 70\% similarity) (Fig. 2B).

Additionally, the HCA dendrogram between NPAA and PA metabolites showed that all detected compounds separated into four clusters. Cluster I included only the monoamine synephrine, which was significantly higher in double-attacked plants compared with other treatments and was clustered separately at the top of the cluster dendrogram (Fig. 2B). Cluster II included four metabolites that showed higher levels in ' $\mathrm{Ca}$. $\mathrm{L}$. asiaticus'-infected plants and were split into two separate groups. The first group included two metabolites (ornithine and $\rho$-aminobenzoic acid) with significantly higher levels only in ' $C a$. L. asiaticus'-infected plants compared with all other treatments, whereas both putrescine and octopamine clustered together in the second group and showed significantly higher levels in both ' $\mathrm{Ca}$. L. asiaticus'-infected and double-attacked plants without any significant differences between them (Fig. 2B). Cluster III included only GABA, which was higher in D. citri-infested plants and was clustered separately in the middle of the dendrogram (Fig. 2B). Cluster IV included three metabolites ( $O$-acetyl serine, pyroglutamic acid, and tyramine), which were decreased significantly in all treatments compared with control (Fig. 2B).

\section{Principal component analysis (PCA) reveals differences in NPAA and PA metabolites.}

The PCA performed using the peak area of individual NPAA and PA metabolites and its associated loading-plot are shown in Figure 3A and B, respectively. The scatter plot obtained from the PCA showed a clear separation among all studied treatments except ' $\mathrm{Ca}$. L. asiaticus'-infected and double-attacked plants, which overlapped each other slightly in the right side of the scatter plot (Fig. 3A). PC1 and PC2 were responsible for $85.11 \%$ of the variation. Furthermore, the loading plot (Fig. 3B) showed that three compounds $(O$-acetyl serine, pyroglutamic acid, and tyramine) were positively correlated with control and four compounds (putrescine, octopamine, ornithine, and $\rho$-aminobenzoic acid) were correlated positively with ' $\mathrm{Ca}$. L. asiaticus'-infected plants. Only one compound was positively correlated with $D$. citri-infested (GABA), and synephrine was correlated with double-attacked plants.

One or both ' $\mathrm{Ca}$. L. asiaticus' infection and

$D$. citri infestation alter the profile of PAAs involved in the GABA shunt and PAs pathways.

For metabolic dissecting of the biosynthetic pathways of NPAAs and PAs, we targeted six PAAs involved in the GABA shunt and PAs pathways in citrus leaves (Fig. 4). L-Cysteine was increased slightly in ' $\mathrm{Ca}$. L. asiaticus'-infected and $\mathrm{D}$. citriinfested treatments, but L-tyrosine remained unchanged in all tested treatments (Fig. 4A and B, respectively). L-Proline, the most abundant amino acid, was significantly increased in all studied treatments, with greater effect for ' $\mathrm{Ca}$. L. asiaticus' infection $(P<0.0001)$ (Fig. 4C), and L-serine also increased significantly in ' $\mathrm{Ca}$. L. asiaticus'-infected leaves $(P<0.0001)$ (Fig. $4 \mathrm{D})$. On the other hand, L-glutamine was reduced significantly in all studied treatments compared with control plants $(P=0.0081)$ (Fig. 4E). Additionally, L-glutamic acid was decreased in the presence of ' $\mathrm{Ca}$. L. asiaticus' (' $\mathrm{Ca}$. L. asiaticus'-infected and double-attacked plants), while it remained at the same level in D. citri-infested plants as in the control $(P<0.0001)$ (Fig. $4 \mathrm{~F})$.

\section{One or both ' $\mathrm{Ca}$. L. asiaticus' infection and D. citri infestation alter the profile of TCA-associated compounds in citrus leaves.}

Four metabolites associated with the TCA cycle were detected in citrus leaves. These compounds included citric acid, 2-ketoglutaric acid, succinic acid, and fumaric acid (Fig. 5). The earliest organic acid in the TCA cycle, i.e., citric acid, was significantly increased in all studied treatments, with greater effect for ' $\mathrm{C} a$. L. asiaticus' infection $(P<0.0001)$ (Fig. 5A). Additionally, both succinic acid and fumaric acid, which appear later in the TCA cycle, were increased significantly in all studied treatments compared with the control plants (Fig. 5B and $\mathrm{C}$, respectively). On the other hand, 2-ketoglutaric acid was significantly reduced in all studied treatments compared with the control plants $(P<0.0001)$ (Fig. 5D).

\section{The citrus genome possesses a putative} GABA permease (GABP).

In silico analysis using the BLASTp tool showed that the Citrus sinensis genome possesses eight sequences with a significant similarity to AtBAT1(NP_565254.1, also known as AtGABP from Arabidopsis thaliana (Supplementary Table S2). The multiple sequence alignment of the amino acid sequence of $A t G A B P$ protein with the eight matched sequences from the citrus genome revealed the similarity and the conserved
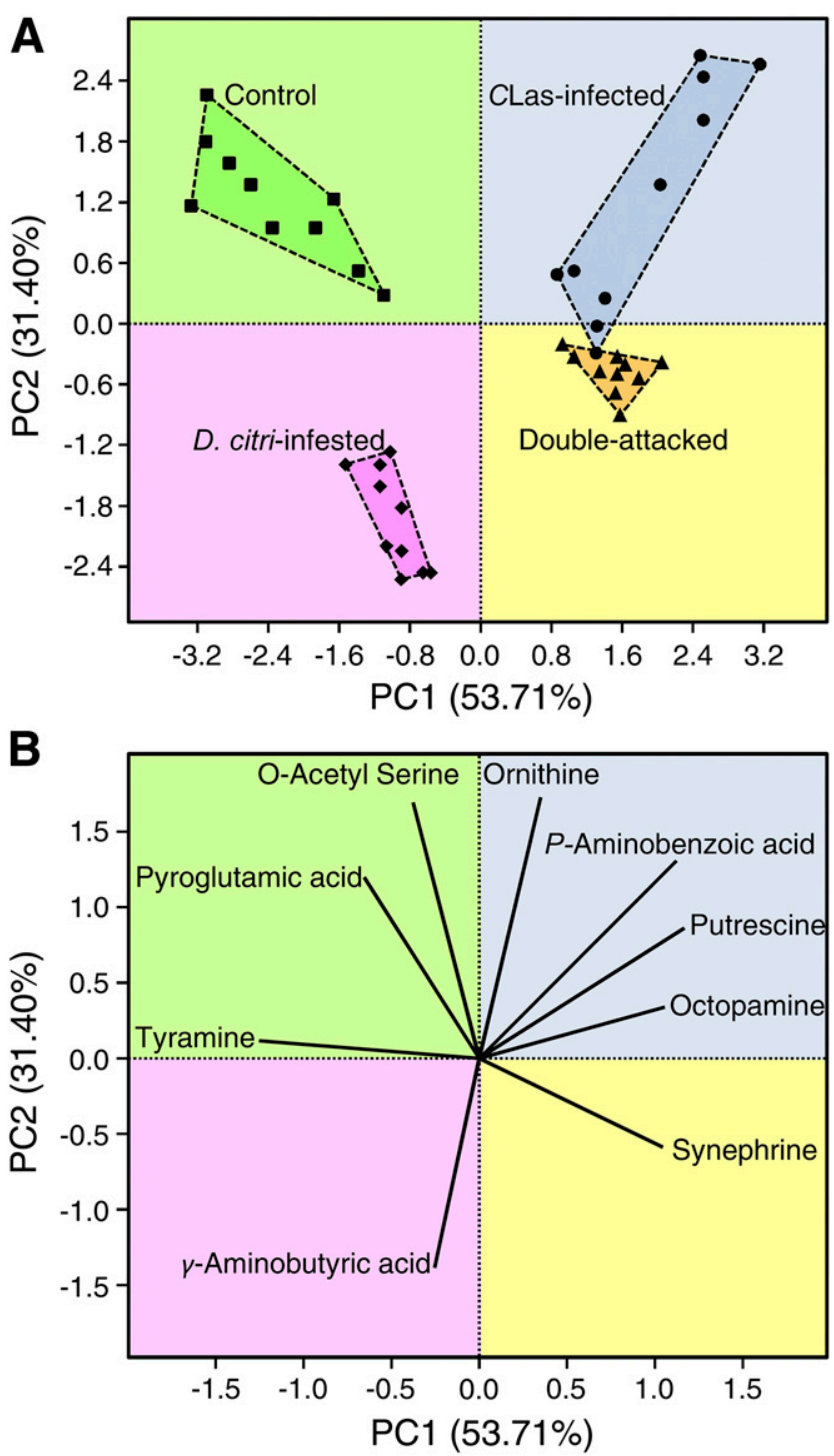

Fig. 3. Principal component analysis (PCA) of individual nonproteinogenic amino acids (NPAAs) and polyamines (PAs) detected in Valencia sweet orange (Citrus sinensis) leaf extracts after either infection with 'Candidatus Liberibacter asiaticus' (CLas), infestation with Diaphorina citri, or both, using gas chromatography-mass spectrometry $(n=10)$. A, PCA scatter plot; B, PCA loading plot. 
sequences of both amino acid permease subfamily and amino acids/polyamines transporter I domains between them (Supplementary Fig. S1). In addition, the phylogenetic analysis using the neighbor-joining method revealed that the predicted amino acid permease BAT1 (CSAAP-BAT1), predicted amino acid permease BAT1-like isoform X1 (CsAAP-BAT1-X; described as $C s G A B P$ in this study), and predicted amino acid permease BAT1-like isoform X2 (CsAAP-BAT1-X2) proteins from $C$. sinensis (XP_006469954.1, XP_006468761.1, and XP_006468762.1, respectively) were phylogenetically closer to GABAP from A. thaliana (AtGABP) than the predicted amino acid permease BAT1-like (XP_006472841.2) and hypothetical proteins CISIN_1g010352mg from C. sinensis (KDO57518.1,
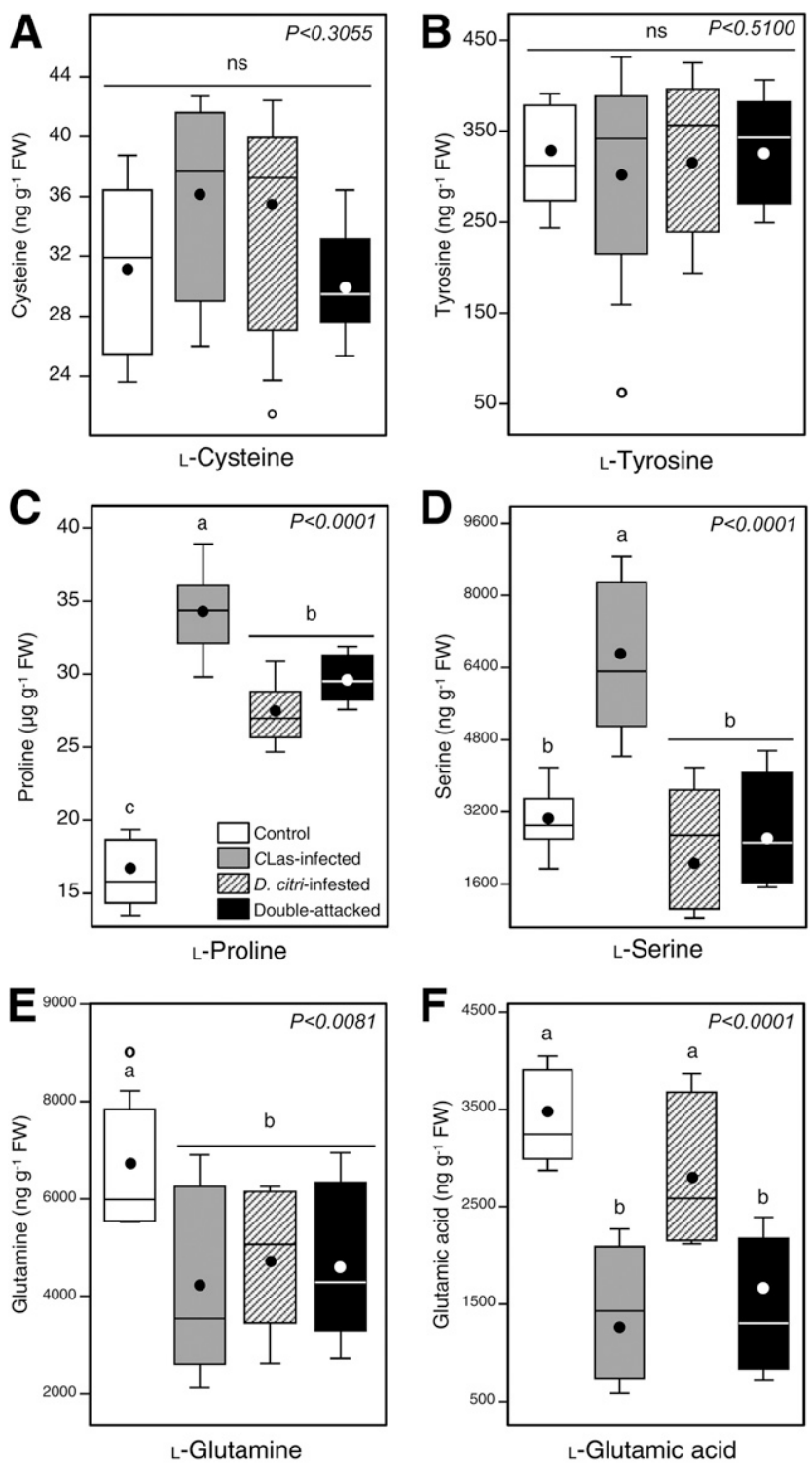

Fig. 4. Concentrations of individual proteinogenic amino acids detected in Valencia sweet orange (Citrus sinensis) leaf extracts after either infection with 'Candidatus Liberibacter asiaticus' (CLas), infestation with Diaphorina citri, or both, using gas chromatography-mass spectrometry ( $n=$ 10). A, L-Cysteine, B, L-tyrosine, C, L-proline, D, L-serine, E, L-glutamine, and F, L-glutamic acid. Horizontal thick lines indicate the medians, black or white dots indicate the means, boxes show the interquartile ranges including 25 to $75 \%$ of the values, whiskers reflect the highest and the lowest value of data. Different letters indicate statistically significant differences among treatments, while "ns" or the same letter signify no significant differences among treatments, using the Tukey-Kramer honestly significant difference test $(P<0.05)$.
KDO57519.1, KDO57520.1, and KDO57522.1), with high bootstrap values as described in Supplementary Figure S2.

Our data showed that $C s G A B P$ protein encoded by the citrus locus LOC102610833 has a relatively high homology with AtGABP protein encoded by the Arabidopsis locus AT2G01170 than other found sequences. Therefore, we focused on this protein for further in silico analysis.

The amino acid sequence of $C s G A B P$ was aligned with the sequences of $A t G A B P$ (Fig. 6A). The alignment showed high similarity and conserved sequences in both amino acid permease subfamily and amino acids/polyamines transporter I domains. Furthermore, the amino acid sequence of $C s G A B P$ had high similarity and conserved sequences when aligned with the $B A T 1$ sequences from other plant species (Supplementary Table S3), including A. thaliana, C. clementina, Brassica napus, Carica papaya, Glycine max, Nicotiana tabacum, Populus trichocarpa, Theobroma cacao, and Vitis vinifera (Supplementary Fig. S3). The homology of CsGABP to proteins from other plant species was more than $90 \%$ identity.

The bioinformatic analysis of amino acid sequences using the InterPro Scan tool and the Phyre2 web portal to predict the conserved domains suggests a high topological similarity between $A t G A B P$ and the CsGABP (Fig. 6B). Both sequences have an amino acid permease subfamily (PF13520, also known as amino acids_permease_2) and amino acids/polyamines transporter I (PIRSF006060, also known as amino acids_ transporter), which contain 12 transmembrane regions that have
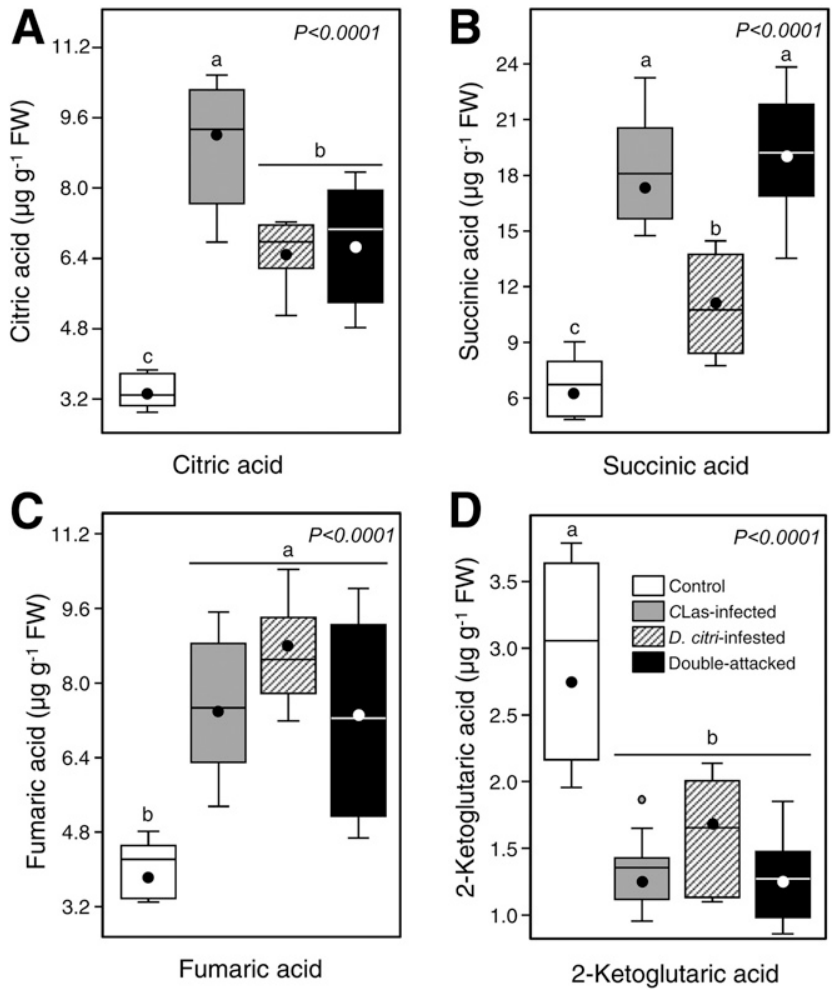

Fig. 5. Concentrations of different individual tricarboxylic acid (TCA)associated compounds detected in Valencia sweet orange (Citrus sinensis) leaf extracts after either infection with 'Candidatus Liberibacter asiaticus' (CLas), infestation with Diaphorina citri, or both, using gas chromatography-mass spectrometry $(n=10)$. A, Citric acid, $\mathbf{B}$, succinic acid, $\mathbf{C}$, fumaric acid, and D, 2-ketoglutaric acid. Horizontal thick lines indicate the medians, black or white dots indicate the means, boxes show the interquartile ranges including 25 to $75 \%$ of the values, whiskers reflect the highest and the lowest value of data. Different letters indicate statistically significant differences among treatments, while " $n s$ " signifies no significant differences among treatments, using the Tukey-Kramer honestly significant difference test $(P<0.05)$. 
been predicted in the sequence to adapt the topology (Fig. 6B; Supplementary Fig. S4). Moreover, the $C s G A B P$ sequence has a conserved site of amino acid permease (PS00218, also known as amino acids_permease_1) (Fig. 6B). Additionally, like other transporters, $C s G A B P$ is predicted to have 12 transmembrane helices with internal $\mathrm{N}$ and $\mathrm{C}$ termini (Fig. 6B).

The predicted three-dimensional (3D) secondary structure of $C s G A B P$ and ligand-binding residues in the conserved motif
A

NP_565254.1 XP_006468761.1

NP_565254.1 XP_006468761.1

NP_565254.1 XP_006468761.1

NP_565254.1 XP_006468761.1

NP_565254.1 XP_006468761.1

NP_565254.1 XP_006468761.1

NP_565254.1 XP_006468761.1

NP_565254.1 XP_006468761.1

NP_565254.1 XP_006468761.1
1 -----MGLGGDOSFVPVMDSGOVRLKELGYKOELKRDLSVFSNFAISFSI ISVLTGITTT 1 MEKLSVP SHIT SNGSVSVDSGHVRLNELGYKQELKRDLSMLSNFAFSES I I SVLTG ITTL 26

56 YNTGLRFGGTVTLVYGWFLAGSFTMCVGLSMAEICSSYPTSGGLYYWSAMLAGPRWAPLA 61 YNTGLNEGGPISLVYGWL IAGAETLFVGSSMAEICSSYPTSGGLYYWSAKLAGPKWAPFA

116 SWMTGWFNIVGQWAVTASVDF SLAQLIQVIVLLSTGGRNGGGYKGSDFVVIGIHGGILFI 121 SWMTGWFNIVGQWAVTTSVDF S LAQMIQVIILLSTGGKNGGGYEASKYVVIAFHGGILIL

176 HALLNSLP ISVLSFIGQLAALWNLLGVLVLMILIPLVSTERAT TKFVFTNFNTDNGLGIT 181 HAIINSLP I ILSFFGQLAAAWNLVGVMVLMI I I SVSTERASAKFVFTHENSDNGDGIN

236 SYAY IFVLGLLMSQYTITGYDASAHMTEETVDADKNGPRGI ISAIGISILFGWGYILGIS 241 SKVYIFVLGLLMSQYTITGYDASAHMTEETKNADRNGPKGIISAIGISIIEGWGYILGIT

296 YAVTDIPSLLSETNNSGGYAIAEIFYLAFKNRFGSGTGGIVCLGVVAVAVFFCGMSSVTS 301 FAVTSIPNLLSEDNDAGGYAIAEIFYLAF KNREGSGVGGIVCLGVVAVAIFFCGMSSVTS

356 NSRMAYAF SRD GAMPMSP LWHKVNSREVP INAVWLSALISFCMALTS LGSIVAF QAMVS I 361 NSRMAYAF SRD GAMPF SS FWHEVNSQD IP INAVWLSAF I SFCMALTYLGSAVAF QAMVS I

416 ATIGLYIAYAIPIILRVTLARNTFVPGPFSLGKYGMVVGWVAVLWVVTISVLFSLPVAYP 421 ATIGLY IAYALP IF FRVTLARK SE IPGPFNLGRYGIVVGWIAVLWVAT I SVLF S LPVAYP

476 ITAETLNYTPVAVAGLVAITLSYWLFSARHWFTGPISNILS 481 ITSDTLNYTPVAVCGLLILTVSAWIFSARHWEKGPITNIAS 151012
B

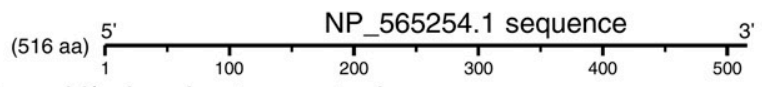

IPR002293: Amino acid/polyamine transporter I

PIRSF006060 (AA_transporter)

PF13520 (AA_permease_2)

Unintegrated signatures

Transmembrane regions

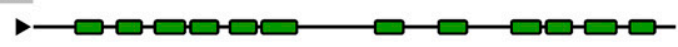

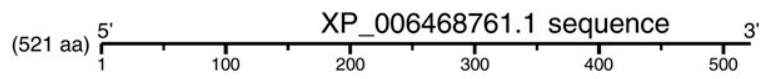

IPR002293: Amino acid/polyamine transporter I

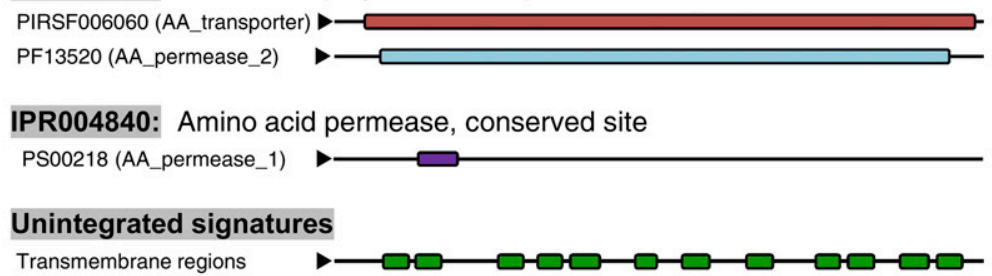

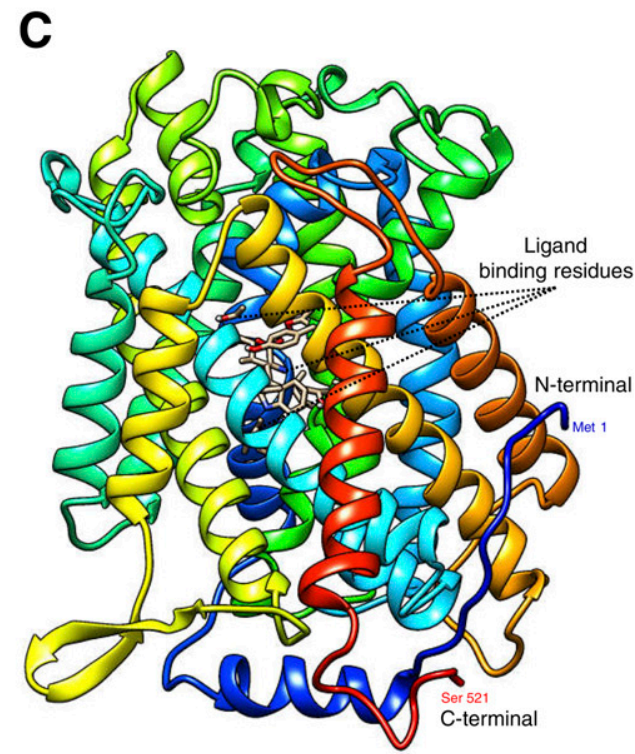

Fig. 6. In silico analysis of $\gamma$-aminobutyric acid (GABA) permease (GABP) of Valencia sweet orange (Citrus sinensis). A, Multiple sequence alignment of GABP amino acid sequences of bidirectional amino acid transporter 1 (AtBAT1) of Arabidopsis thaliana (NP_565254.1, also known as AtGABP) and the predicted amino acid permease BAT1-like isoform X1 of C. sinensis (XP_006468761.1, also known as CsGABP). Conserved amino acids are indicated with black shading and those with high similarity score are in gray. The lines below the sequences mark the conserved domains of AtGABP for the amino acid permease subfamily and amino acid/polyamines transporter I, respectively. Whiskers reflect the start and the end of each domain. Numbers before and after whiskers denote amino acid residue number for the start and end, respectively. B, Protein functional and conserved domains analysis of AtGABP (NP_565254.1) and CsGABP (XP_006468761.1), using InterPro Scan. C, The predicted three-dimensional secondary structure model of CsGABP (XP_006468761.1), with 87\% similarity, with $100.0 \%$ confidence, by the single highest-scoring template with the structure of glutamate-GABA antiporter GadC (4DJI) from Escherichia coli (strain K12) in the Protein Data Bank. All bioinformatic analyses were carried out using the available data of the C. sinensis cv. Valencia v2.0 genome from chromosome level HZAU assembly (Xu et al. 2013) in the Citrus Genome database and recent data on the National Center for Biotechnology Information's GenBank website. 
site are shown in Figure 6C. The 3D secondary structure model of $C s G A B P$ protein showed that about $87 \%$ of the sequence (452 residues of 521 amino acids) have been modeled with $100.0 \%$ confidence by the single highest-scoring template with the structure of glutamate-GABA antiporter GadC (4DJI) from Escherichia coli (strain K12) in the Protein Data Bank (PDB). Furthermore, the predicted normalized B-factor by ResQ, obtained by the I-TASSER server and associated with the predicted 3D model, showed that most of the residues are relatively more stable in the structure, in which most of them had negative normalized B-factor values.

Taken together, these results suggest that the citrus genome contains the locus XM_006468698.3 (1,927 bp), which encodes for a putative $C$. sinensis amino acid permease BAT1like-protein (LOC102610833), transcript variant X1 (Cs2g13200.1, also known $C s G A B P$ ). This protein is conserved and contains 521 amino acids. The amino acid sequence and predicted topology of $C S G A B P$ resemble those of the mitochondrial AtGABP of A. thaliana.

' $\mathrm{Ca}$. L. asiaticus' and $\mathrm{D}$. citri alter the expression of genes implicated in the GABA shunt cycle and the PAs pathway.

In addition to $C s G A B P$, we investigated the transcript levels of 32 genes involved in the GABA shunt and the PAs biosynthetic pathway in Valencia sweet orange leaves (Fig. 7). Gene expression data was normalized, using four reference housekeeping genes (Supplementary Table S4) that previously showed high stability for transcript normalization in citrus under biotic stress (Mafra et al. 2012; Wei et al. 2014). The normalized expression levels using the four housekeeping genes were similar to each other (data not shown). Generally, the expression levels of all investigated genes increased after either or both infection with ' $\mathrm{Ca}$. L. asiaticus' and infestation with D. citri.

Comparisons of the relative fold change of the $C s G A B P$ gene, which connects the TCA cycle and GABA shunt, showed that $C s G A B P$ was up-regulated in all treatments compared with control plants (Fig. 7A). CsGABP was expressed at higher levels (up to fourfold) in ' $C a$. L. asiaticus'-infected and D. citri-infested plants, followed by double-attacked plants (about 2.2-fold) (Fig. 7A). In addition, the differences in relative expression levels of the 32 investigated genes involved in the GABA shunt and PAs biosynthetic pathways were visualized as a heat map combined with a standardized two-way HCA (Fig. 7B). The total HCA dendrogram among treatments (Fig. $7 \mathrm{~B}$, bottom) showed that the transcript levels of ' $\mathrm{Ca}$. L. asiaticus'-infected plants were closer to the profile of $D$. citriinfested ones (approximately 90\% similarity) than doubleattacked plants (just less than 85\% similarity) (Fig. 7B).

Furthermore, the total HCA dendrogram among the investigated genes showed that all 32 genes separated into three distinct clusters. Cluster I includes 19 genes that were expressed at higher levels after D. citri infestation. Most of these genes are involved in the GABA shunt, PAs biosynthesis, and the glutamate pathway (Fig. 7B). Cluster II includes 10 genes with higher levels in ' $\mathrm{Ca}$. L. asiaticus'-infected plants than other treatments and that were split into two separate clusters (cluster II-1 and cluster II-2). Interestingly, cluster II contains the predicted $C$. sinensis serine acetyltransferase 1 chloroplastic-like (CSSAT1, XM_006474855.2) and predicted C. sinensis serine acetyltransferase 5 (CsSAT5, XM_ 006475346.2). These two genes are involved in $O$-acetyl serine biosynthesis. Furthermore, cluster II also contains the predicted C. sinensis tyrosine decarboxylase 1 (CsTDC1, XM_ 006479363.2), the only gene in the synephrine biosynthetic pathway that has been isolated and cloned from a plant source (Fig. 7B). Cluster III includes only three genes that showed higher levels in the presence of one or both ' $\mathrm{Ca}$. L. asiaticus' and D. citri compared with control plants (Fig. 7B). Interestingly, cluster III contains the predicted $C$. sinensis polyamine oxidase 1 gene (CsPAO, XM_006482854.1) that catalyzes the oxidation of spermidine to GABA, resulting in accumulation of $\mathrm{H}_{2} \mathrm{O}_{2}$ within the apoplast. According to these findings, the gene expression results support our findings from the GC-MS work.

\section{DISCUSSION}

The TCA cycle is a commonly ubiquitous metabolic hub for ensuring availability of the required cellular energy via the metabolism of carbohydrates, lipids, and amino acids under aerobic conditions (Araújo et al. 2012; Sweetlove et al. 2010). Due to its importance in energy metabolism and other biological processes, the TCA cycle has been intensively investigated in many species belonging to both prokaryotes and eukaryotes (Blank and Sauer 2004; Bolton 2009; Cavalcanti et al. 2014; Xiong et al. 2014). However, we are just beginning to better understand the roles of the TCA cycle in the HLB pathosystem. Our previous studies showed that ' $\mathrm{Ca}$. L. asiaticus' manipulates the TCA cycle of its vector, $D$. citri, for its benefit (Killiny and Jones 2018; Killiny et al. 2017a, 2018b; Lu and Killiny 2017), by inducing metabolic changes that can help meet its nutritional needs or to neutralize the host defense responses. Recently, it has been shown that citrate and other TCA cycle-associated compounds are necessary as energy sources for $L$. crescens growth on chemically defined media (CruzMunoz et al. 2018). However, more studies are required to explore the roles of the TCA cycle metabolic pathway in fulfilling the nutritional needs of $\mathrm{D}$. citri and ' $\mathrm{Ca}$. L. asiaticus'.

Although the mitochondria possess all enzymes for an intact TCA cycle, several lines of evidence from metabolic networking studies suggest that the TCA cycle in plants may work as independent or partial sets of reactions based on the metabolic and physiological needs of the cells (Sweetlove et al. 2010). In the current study, we found that the infection with ' $C a$. L. asiaticus' and the infestation with $D$. citri altered the TCA cycle of the host plant. For instance, both ' $\mathrm{Ca}$. L. asiaticus' infection and D. citri infestation reduced the $\alpha$-ketoglutarate and induced the accumulation of succinate, fumarate, and citrate. We hypothesize that citrus plants might have one or more additional or alternative pathways that may contribute to this flux toward succinate rather than as an intact TCA cycle. These alternative fluxes might occur in citrus under specific abiotic and biotic stresses such as ' $\mathrm{C} a$. L. asiaticus' infection and $D$. citri infestation. Both the GABA shunt and PAs pathway are potential candidates for these alternative pathways (from $\alpha$-ketoglutarate to succinate) and they might be enhanced after the major flux from $\alpha$-ketoglutarate to glutamate biosynthesis.

The GABA shunt is the first potential alternative route that could provide a connection between $\alpha$-ketoglutarate and succinate outside the TCA cycle (Fait et al. 2008; Xiong et al. 2014). It has been suggested that GABA contributes to citrate degradation, and the GABA shunt is involved in the central carbon and nitrogen metabolism (Cercós et al. 2006; Michaeli et al. 2011). The GABA shunt is a short pathway conserved in many prokaryotic and eukaryotic species that bypasses two steps of 2-oxoglutarate-to-succinate conversion outside the TCA cycle (Bouché and Fromm 2004; Xiong et al. 2014). The biological function of the GABA shunt in plants is still not well-understood (Xiong et al. 2014). However, GABA plays a key role in plant growth and development (Palanivelu et al. 2003), oxidative stress sensing and tolerance (Bouché et al. 2003), quorum-sensing signaling in plant-bacteria systems (Chevrot et al. 2006), extracellular signaling (Roberts 2007), 
plant defense response (Bolton 2009), and carbon/nitrogen balancing. In addition, it has been reported that exogenous GABA supplementation greatly enhanced citrate and amino acid accumulation (including glutamate, alanine, serine, aspartate, and proline) and improved the fruit quality of citrus (Sheng et al. 2017).

In higher plants, the GABA shunt is linked with the TCA cycle through the production of succinate, using either $\alpha$-ketoglutarate or glutamate as precursors (Bolton 2009; Bouché and Fromm 2004; Shelp et al. 1999). In addition, it has
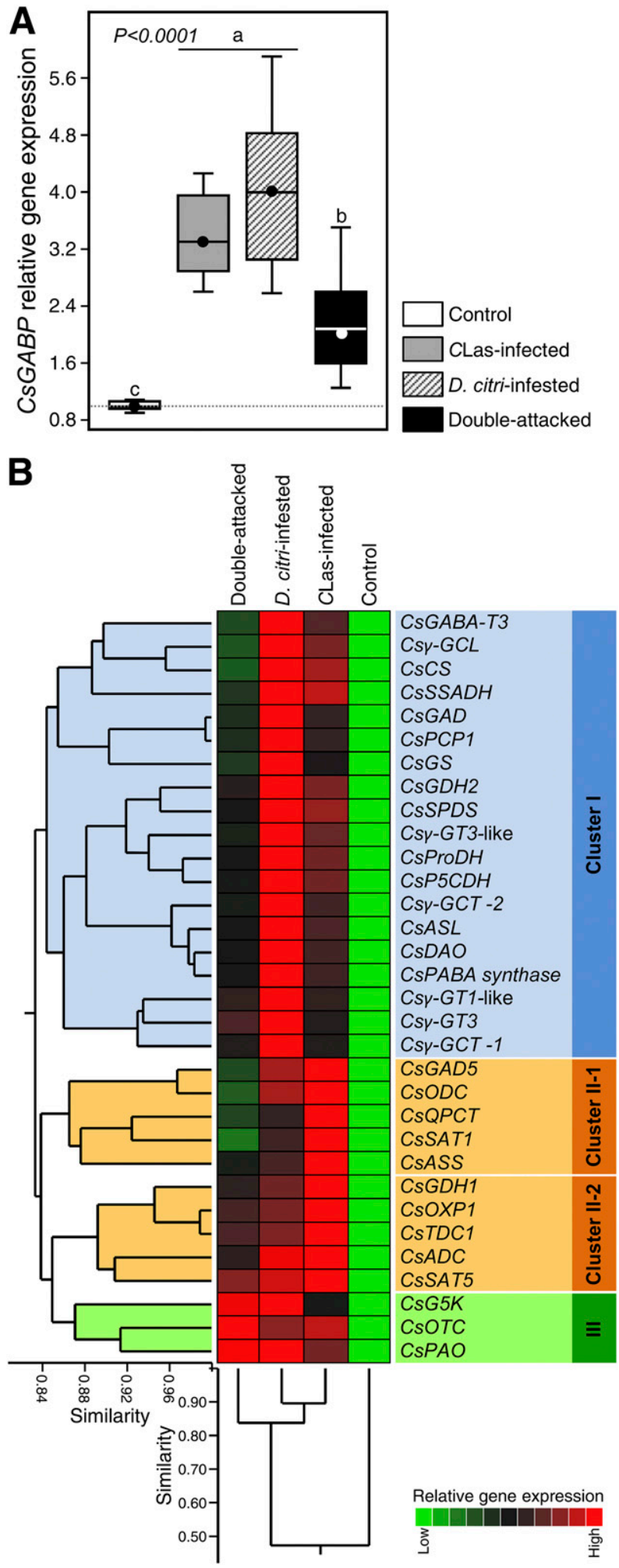

been shown that citric acid catabolism is connected to the GABA shunt in citrus fruits (Hussain et al. 2017; Sheng et al. 2017). Briefly, citric acid is catalyzed in the cytosol to $\alpha$-ketoglutaric acid, then to glutamic acid, then to GABA via isocitrate dehydrogenase, aspartate aminotransferase (or alanine aminotransferase), and glutamate decarboxylase (GAD) activities, respectively (Bown and Shelp 1997; Cercós et al. 2006). Subsequently, GABA is transported from the cytosol to the mitochondria using mitochondrial GABP and, then, is converted to succinic semialdehyde, paving the way for reentry of succinate to the TCA cycle by GABA aminotransferase $(G A B A-T)$ and succinate semialdehyde dehydrogenase $(S S A D H)$, respectively (Cercós et al. 2006; Fait et al. 2008). Interestingly, our findings showed that both ' $\mathrm{Ca}$. L. asiaticus' infection and $D$. citri infestation enhanced the succinate level. This might be due to the catabolism of GABA to succinate directly or through the conversion to succinate-semialdehyde by $C s G A B A-T$, then to succinate, using $C s S S A D H$ in sequential reactions. Thus, our findings indicate that succinate could be biosynthesized via the GABA shunt rather than from $\alpha$-ketoglutarate, which supports the previous study (Shelp et al. 1999).

Other enzymes involved in this pathway include glutamate dehydrogenase, glutamate synthase, $\gamma$-glutamylcysteine synthetase, and glutamate 5-kinase (Shelp et al. 1999, 2017). Our findings showed that these enzymes were expressed at higher levels upon ' $C a$. L. asiaticus' infection and $D$. citri infestation, indicating that the reduction of $\alpha$-ketoglutarate could be due to the acceleration of its conversion to glutamate (L-glutamine and L-glutamic acid) or pyroglutamic acid, resulting in an accumulation of GABA by these enzymes. Moreover, our finding showed that L-glutamine, L-glutamic acid, and pyroglutamic acid were also deceased after ' $\mathrm{Ca}$. L. asiaticus' infection and $D$. citri infestation. Taken together, these findings support the hypothesis that ' $C a$. L. asiaticus' and $D$. citri accelerate the conversion of $\alpha$-ketoglutarate to glutamate (L-glutamine, L-glutamic acid, and pyroglutamic acid) and also accelerate the conversion of glutamate to GABA, causing an accumulation of GABA in the cytosol.

Fig. 7. Relative gene expression of genes involved in the $\gamma$-aminobutyric acid (GABA) shunt and other polyamines (PAs) biosynthetic pathways in Valencia sweet orange (Citrus sinensis) leaves after either infection with 'Candidatus Liberibacter asiaticus', infestation with Diaphorina citri, or both. A, Relative gene expression of the predicted amino acid permease BAT1-like isoform X1 of C. sinensis (XP 006468761.1, also known as CsGABP). Horizontal thick lines indicate the medians, black or white dots indicate the means, boxes show the interquartile ranges including 25 to $75 \%$ of the values, whiskers reflect the highest and the lowest value of data. Different letters indicate statistically significant differences among treatments, while the same letter signifies no significant differences among treatments, using the Tukey-Kramer honestly significant difference test $(P<0.05)$. B, Two-way hierarchical cluster analysis $(\mathrm{HCA})$ and its associated heat map diagram of differential standardized gene expression patterns of 32 genes involved in the GABA shunt and PAs biosynthetic pathway. Rows represent the genes while columns represent different treatments. Treatments and genes were organized using HCA based on similarities in autoscaled values and correlations, respectively, with $95 \%$ confidence between groups from the discriminant function analysis, to construct the similarity dendrograms. Gene expressions were normalized using four housekeeping genes (EF1, F-box, GAPC1, and SAND), which previously showed high stability for transcript normalization in different citrus organs under biotic stress (Mafra et al. 2012; Wei et al. 2014). The changes in gene expression were analyzed with the $2^{-\Delta \Delta C T}$ method. Samples were analyzed in triplicate for each technical replicate (five biological replicates, two technical replicates each per treatment; $n=30$ ). The listed genes were assembled based on the available data of the $C$. sinensis cv. Valencia v2.0 genome from chromosome level HZAU assembly (Xu et al. 2013) in the Citrus Genome database and recent data on the National Center for Biotechnology Information's GenBank website. 
However, GABA is mainly biosynthesized from glutamate by GAD, which is a key enzyme in this pathway (Bouché and Fromm 2004; Fait et al. 2008; Liu et al. 2014). We identified two GAD genes in the $C$. sinensis genome, glutamate decarboxylase-like (CsGAD; NM_001288909.1) and glutamate decarboxylase 5-like (CsGAD5; XM_006478039.2). Both genes are upregulated after ' $\mathrm{Ca}$. L. asiaticus' infection and D. citri infestation. The HCA and gene expression results indicated that CsGAD was mainly expressed in $D$. citri-infested plants and CsGAD5 was mainly expressed in 'Ca. L. asiaticus'infected plants. Taken together, the gene expression results suggest that different stressors could induce different CsGAD transcripts. The previous study on CsGADs of citrus plants showed that CsGAD1 and CsGAD2 transcripts were mainly expressed in flowers and in fruit juice sacs, respectively (Liu et al. 2014).

The functional connection between the GABA shunt and the TCA cycle in higher plants and its role in primary carbon metabolism has been reported previously (Fait et al. 2008; Michaeli et al. 2011; Rocha et al. 2010). However, the linkage between TCA cycle and GABA shunt in citrus is unknown. Recently, Michaeli et al. (2011) reported that a mitochondrial $G A B P$ connects the GABA shunt and TCA cycle and plays a key role in normal carbon metabolism in A. thaliana (Michaeli et al. 2011). The $C$. sinensis genome could encodes for a putative amino acid permease BAT1-like-protein, transcript variant $\mathrm{X} 1$. This protein was described in the current study as $C s G A B P$, indicating its predicted GABP activity. $C s G A B P$ may play a key role in connecting the GABA shunt and the TCA cycle, acting as a GABA transporter, and may be essential for primary carbon metabolism. Its function as GABA transporter was suggested due to its high gene expression levels upon ' $\mathrm{Ca}$. L. asiaticus' infection and D. citri infestation. The accumulated GABA in the cytosol needs to be transported into the mitochondria, where it is converted to succinate and integrated into the TCA cycle (Fait et al. 2008; Michaeli et al. 2011). CsGABP could play an important role in this process, similar to the role of AtGABP in Arabidopsis (Michaeli et al. 2011). However, further studies are required to clarify the functional or regulatory roles of $C s G A B P$ in citrus.

The second suggested pathway that could be involved in the enrichment of the TCA cycle is the PAs biosynthetic pathway. We showed that ' $C a$. L. asiaticus' infection induced the biosynthesis of PAs, leading to an accumulation of ornithine and putrescine. In plants, putrescine is synthesized through two different routes, from arginine, using arginine decarboxylase (ADC), or from ornithine mediated by ornithine decarboxylase (ODC) (Moschou et al. 2012). In this study, ornithine was increased after ' $\mathrm{Ca}$. L. asiaticus' infection. In addition, arginine was detected only in ' $\mathrm{Ca}$. L. asiaticus'-infected plants (data not shown). Moreover, both $C s A D C$ and $C s O D C$ were expressed at higher levels in ' $\mathrm{Ca}$. L. asiaticus'-infected plants, indicating that both putrescine biosynthetic pathways are jointly working to enhance the PAs content. However, the role of PAs in HLB pathosystem is poorly understood. We believe that although PAs might play a role in citrus defense against ' $\mathrm{Ca}$. L. asiaticus' and its vector, they could also be involved in disease symptom development via the production of $\mathrm{H}_{2} \mathrm{O}_{2}$. Briefly, diamine oxidase (DAO) and polyamine oxidase (PAO) catalyze the oxidation of putrescine and spermidine, respectively, to produce GABA and resulting in accumulation of $\mathrm{H}_{2} \mathrm{O}_{2}$ within the apoplast (Walters 2000, 2003a).

The accumulation of $\mathrm{H}_{2} \mathrm{O}_{2}$ in citrus plants after infection with ' $C a$. L. asiaticus' has been reported previously (Pitino et al. 2017). In our study, D-amino acid oxidase PA4548 (also known as $C s D A O)$ and PAO 1 (CsPAO) were expressed at higher levels in ' $\mathrm{C} a$. L. asiaticus'-infected plants, which agreed with this common feature of pathogen-host interaction in many previous studies (Walters 2000, 2003a). Thus, we hypothesize that the induction of PAs and their catabolic genes (CsDAO and CsPAO) are correlated with the accumulation of GABA and $\mathrm{H}_{2} \mathrm{O}_{2}$, which are working as a response to HLB to create an incompatible interaction. Nevertheless, the alteration in DAO and PAO and the accumulation of $\mathrm{H}_{2} \mathrm{O}_{2}$ have to occur in the appropriate location of the plant to be effective in the creation of incompatible interactions between the host and the pathogen (Walters 2003a). Interestingly, ' $\mathrm{Ca}$. L. asiaticus' could survive the toxic effects of accumulated $\mathrm{H}_{2} \mathrm{O}_{2}$ using its own peroxidase (Pitino et al. 2017). The detoxification system of citrus plants, however, might not be sufficient to reduce the high $\mathrm{H}_{2} \mathrm{O}_{2}$ levels, which may eventually become toxic to the leaf tissue and cause the characteristic blotchy mottle symptoms that appear after 'Ca. L. asiaticus' infection (Pitino et al. 2017).

In addition, the PAs biosynthesis pathway could be involved in the enrichment of the TCA cycle directly through the production of the dicarboxylic acid fumarate. Fumarate could be produced during the conversion of citrulline to argininosuccinate by argininosuccinate synthase (ASS), then to the amino acid arginine, using argininosuccinate lyase (ASL, also known as argininosuccinase), which splits argininosuccinate to release fumarate and arginine (Haines et al. 2011). Our findings showed that both $C s A S S$ and $C s A S L$ were upregulated after ' $C a$. L. asiaticus' infection, which resulted in the accumulation of arginine (detected only in ' $\mathrm{Ca}$. L. asiaticus'-infected plants). Together, this might be enhancing the TCA cycle intermediate fumarate directly (Fig. 8). Similar induction of fumarate was observed during the necrotrophic phase of rice blast fungus Magnaporthe grisea (Parker et al. 2009) and the maize anthracnose fungus Colletotrichum graminicola (Voll et al. 2011).

Furthermore, the phenolic monoamine synephrine (Fig. 8) and its precursor, octopamine, are compounds that might be involved in the defense response against HLB. Synephrine was reported previously in several citrus species and cultivars (Arbo et al. 2008; Dragull et al. 2008). In citrus, it is believed that synephrine is biosynthesized from tyrosine via a pathway involving tyramine, $N$-methyltyramine, then to synephrine using tyrosine decarboxylase (TDC), tyramine $N$-methyltransferase (TNMT), and an unidentified N-methyl-tyramine- $\beta$-hydroxylase (NMT $\beta$ H), respectively (Wheaton and Stewart 1969; Dragull et al. 2008; Bartley et al. 2010). In animals, synephrine is biosynthesized from tyrosine through tyramine, octopamine, then to synephrine using TDC, tyramine $\beta$-hydroxylase $(\mathrm{T} \beta \mathrm{H})$ and phenylethanolamine $N$-methyltransferase (PNMT), respectively (Wheaton and Stewart 1969). Although octopamine is not as important an intermediate in plants as in animals, our results showed that the octopamine level was elevated in the presence of 'Ca. L. asiaticus'. Although the transcript level of CsTDC was increased in all studied treatments compared with control plants, tyramine level was decreased in 'Ca. L. asiaticus'-infected plants but not other treatments. This might be due to the acceleration of the conversion of tyramine to synephrine via $N$-methyltyramine (most common in plants) or via octopamine (most common in animals). Because only the TDC has been isolated and cloned from plants, while other enzymes (TNMT and NMT $\beta \mathrm{H}$ in plants and T $\beta \mathrm{H}$ and PNMT in animals) have not yet been cloned (Bartley et al. 2010), our image about this pathway (synephrine-polyamine pathway) and the reasons behind tyramine reduction is not fully understood and might require further studies. Taken together, our findings indicate that CsTDC is involved in synephrine biosynthesis in citrus, in agreement with Bartley et al. (2010).

To summarize our findings, a hypothetical model for the connection between the TCA cycle, PAs pathway, and GABA shunt in citrus was suggested and presented in Figure 8. In this 


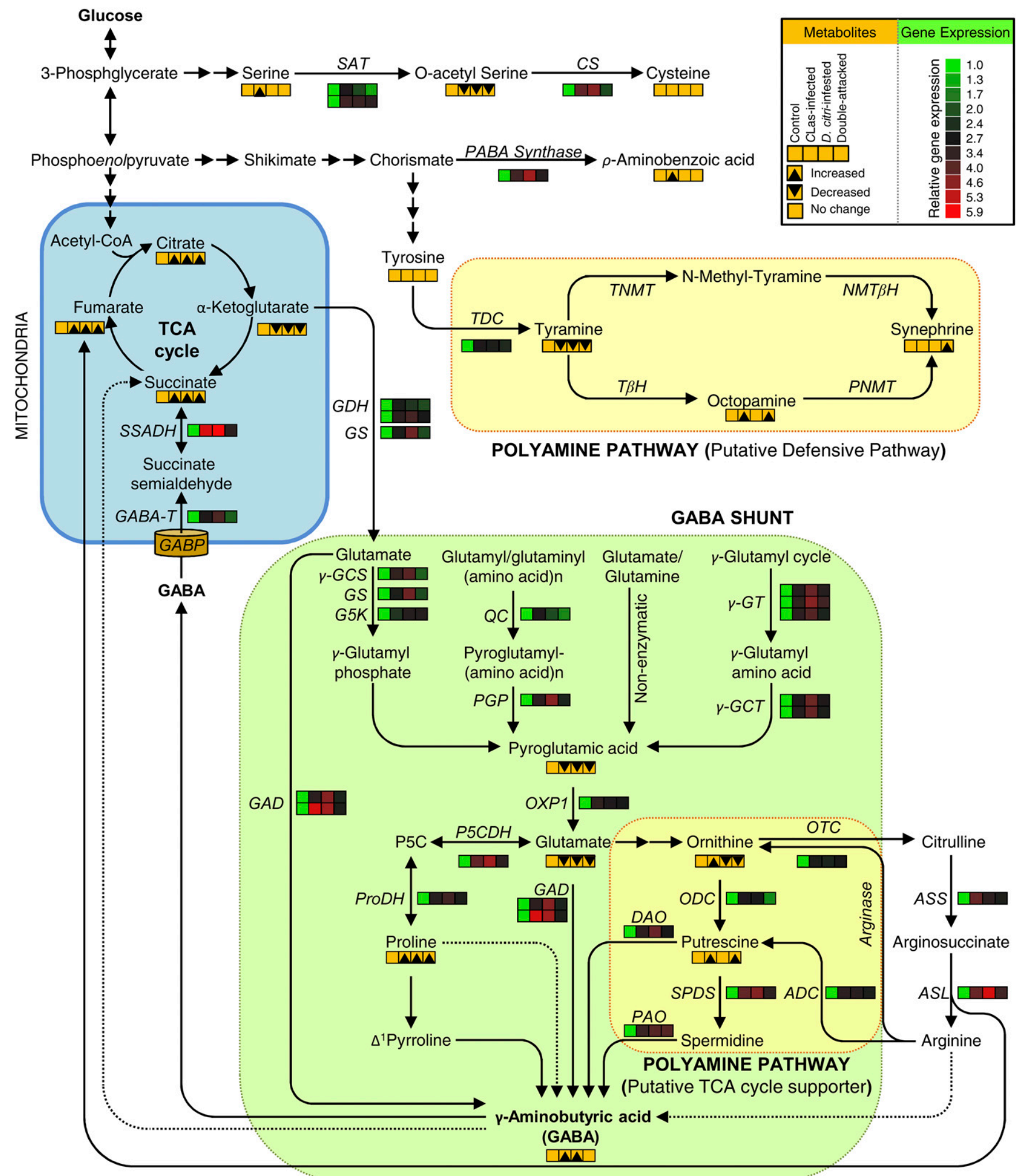

Fig. 8. Hypothetical model of the effect of either infection with 'Candidatus Liberibacter asiaticus', the infestation with Diaphorina citri, or both on the proteinogenic amino acids, nonproteinogenic amino acids, polyamines (PAs), and tricarboxylic acid (TCA)-associated compounds of Valencia sweet orange (Citrus sinensis) leaves. Briefly, we suggest that one or both ' $C a$. L. asiaticus' infection and $D$. citri infestation augment the TCA cycle of the citrus plant indirectly via the $\gamma$-aminobutyric acid (GABA) shunt cycle and PAs pathway. ' $C a$. L. asiaticus' infection, $\mathrm{D}$. citri infestation, or both might accelerate the conversion of $\alpha$-ketoglutarate to glutamate, which eventually results in the accumulation of GABA. Subsequently, GABA is transported from the cytosol to the mitochondria via GABA permease (GABP), to support the accumulation of succinate directly or indirectly. These findings indicate that succinate is biosynthesized predominantly via the GABA shunt rather than from $\alpha$-ketoglutarate. Another pathway that could be involved in the enrichment of the TCA cycle is the PAs biosynthetic pathway. The PAs pathway might be connected directly to the TCA cycle, through the production of the dicarboxylic acid fumarate during the catabolism of argininosuccinate to arginine using argininosuccinate lyase (ASL), or indirectly, via the enhancement of GABA shunt. Taken together, we suggest a functional connection between the GABA shunt and the TCA cycle in citrus plants via GABP. Solid lines with arrows signify positive reaction and dotted lines with arrows represent hypothetical mechanisms or uncharacterized elements. 
model, we proposed that ' $\mathrm{Ca}$. L. asiaticus' and its insect vector, $D$. citri, augments the TCA cycle of citrus plant indirectly via the GABA shunt cycle and PAs pathways to increase the flux toward succinate, thereby increasing the availability of fumarate and citrate above that provided by the TCA cycle alone. Briefly, ' $C a$. L. asiaticus' infection might accelerate the conversion of $\alpha$-ketoglutarate to glutamate, then to GABA, which results in an accumulation of GABA in the cytosol. Subsequently, GABA is transported from the cytosol to the mitochondria, using the GABP, to support the accumulation of succinate directly or indirectly. Another pathway that could be involved in the enrichment of the TCA cycle is the PAs biosynthesis pathway. The PAs pathway might be connected directly to the TCA cycle through the production of the dicarboxylic acid fumarate during the catabolism of argininosuccinate to arginine, using ASL (also known as argininosuccinase), or indirectly via the enhancement of GABA shunt. In addition, $\alpha$-ketoglutarate is involved in many other metabolic pathways, such as the amino acid proline biosynthesis. Proline is biosynthetically derived from the glutamate via the 81-pyrroline-5-carboxylate. In Figure 8, we hypothesize that there might be a connection between proline and GABA to enhance the GABA content. However, we believe that this connection or hypothetical route between proline and GABA requires further investigation to be confirmed

The importance of this study is not only to provide a clue about the role of the TCA cycle, GABA shunt, and PAs biosynthetic pathway in citrus response against HLB but to possibly underscore the functional connection between the GABA shunt and the TCA cycle. This connection was not reported previously in citrus. Furthermore, this study may provide some insights into the understanding of the nutritional needs of ' $\mathrm{Ca}$. L. asiaticus', which may lead to successful culturing of this bacterium. Finally, understanding the metabolic changes in citrus after ' $C a$. L. asiaticus' infection or $D$. citri infestation could lead to a comprehensive picture of defense responses to HLB in citrus, which is critical to finding novel and sustainable management strategies for HLB.

\section{MATERIALS AND METHODS}

\section{Plant materials and growth conditions.}

Valencia sweet orange (Citrus sinensis (L.) Osbeck) trees, 80 $\pm 5 \mathrm{~cm}$ tall and around 18 months old at the time of sampling, were used for this study. Trees were maintained in an approved United States Department of Agriculture Animal and Plant Health Inspection Service/Centers for Disease Control and Prevention secured greenhouse, at $28 \pm 2^{\circ} \mathrm{C}$, with $65 \pm 5 \%$ relative humidity and a $16-\mathrm{h}$ light and 8 -h dark photoperiod. The facility is located at the Citrus Research and Education Center, University of Florida, Lake Alfred. Trees (caged or noncaged) were randomly placed in the greenhouse, were irrigated twice weekly, and were fertilized monthly with 20:10: 20 NPK water-soluble fertilizer. In this study, four treatments were tested, including: control (healthy), ' $C a$. L. asiaticus'infected, $D$. citri-infested, and double-attacked trees (' $C a$. L. asiaticus'-infected and D. citri-infested together). For each treatment, ten biological replicates were analyzed (two technical replicates for each). The technical replicates were used only to test the reproducibility and variability in the extraction protocol and GC-MS machine but were not used for statistical analysis. Since our method showed a high reproducibility, the values of each pair of technical replicates were very close to each other.

To obtain the ' $\mathrm{Ca}$. L. asiaticus'-infected trees, 10-month-old, HLB-free Valencia trees were graft-inoculated with budwoods from a polymerase chain reaction (PCR)-positive HLB-infected citrus tree and were maintained in the same conditions as described above. Upon initial symptom development, approximately 7 months later, the infection with ' $\mathrm{Ca}$. L. asiaticus' was confirmed by PCR (Tatineni et al. 2008). To obtain both D. citri-infested and double-attacked plants, 16 month-old healthy or ' $C a$. L. asiaticus'-infected trees, with new growth flush, were exposed to 100 healthy adult psyllids (PCRnegative; previously reared on Bergera koenegii, nonhost for ' $C a$. L. asiaticus') per plant and caged individually, using insect rearing cages $(60 \times 60 \times 90 \mathrm{~cm})$ and were maintained in the growth room under the same conditions as described above. One month later, both $D$. citri-infested and double-attacked plants were cleaned from all $D$. citri stages.

For sampling, three leaves were collected per tree from different positions and different ages, juvenile leaves from the top, intermediate-aged leaves (fully expanded but not hardened) from the middle, and mature leaves (deep green and hardened) from the lower part of the plant. The collected leaves were chopped, were mixed together, and were immediately kept on ice. Plant materials were kept at $-80^{\circ} \mathrm{C}$ until further analysis.

\section{Analysis of citrus leaf NPAAs, Pas, and TCA-associated compounds.}

Extraction of citrus leaf NPAAs, PAs and TCA compounds. Citrus leaf NPAAs, Pas, and TCA-associated compounds (2ketoglutaric acid, succinic acid, fumaric acid, and citric acid) were extracted from frozen tissue using methanol $80 \%$ containing $0.1 \% \mathrm{HCl} 6 \mathrm{~N}$, as described in our previous studies (Killiny and Nehela 2017a; Nehela et al. 2016). The extraction was repeated three times from the same biological sample and the supernatants were combined. The collected supernatant was concentrated to $50 \mu \mathrm{l}$ under a nitrogen stream and was stored at $-80^{\circ} \mathrm{C}$ for further work.

MCF derivatization of citrus leaf metabolites. Before derivatization, each sample was spiked with a $5 \mu$ l aliquot of 200ppm heptadecanoic acid, which is not found in citrus leaves, as an internal standard. Citrus leaf NPAAs, PAs, and TCAassociated compounds were derivatized with MCF following the protocol of Hijaz and Killiny (2014), with slight modifications as described by (Killiny and Nehela 2017a). Briefly, $50 \mu \mathrm{l}$ of the methanol extract of citrus leaves was derivatized twice with $20 \mu \mathrm{l}$ of MCF, then extracted into chloroform. After derivatization, about $40 \mu \mathrm{l}$ of the organic layer was transferred to a new insert tube. Then, a few milligrams of sodium sulfate (two to three crystals) were added to dry the organic layer. For GC-MS analysis, $1 \mu \mathrm{l}$ was injected into a GC-MS running in the full scan mode for NPAAs, PAs, and TCA-associated compounds analysis.

GC-MS analyses of citrus leaf NPAAs, PAs, and TCA compounds. All derivatized samples and standards were analyzed using a Clarus 680 GC-MS system (Perkin Elmer) fitted with a ZB-5MS GC column (5\% phenyl-arylene $95 \%$ dimethylpolysiloxane; low bleed, $30 \mathrm{~m} \times 0.25 \mathrm{~mm} \times 0.25 \mu \mathrm{m}$ film thickness [Phenomenex]). Helium gas was used as the carrier gas with a flow rate of $1 \mathrm{ml} \mathrm{min}{ }^{-1}$. The GC thermo program, MS ion identification, and GC-MS chromatogram analysis were performed according to Killiny and Nehela (2017a).

Identification of citrus leaf NPAAs, PAs and TCA compounds. All studied NPAAs, Pas, and TCA-associated compounds were first identified by comparing their mass spectra with library entries of NIST 2011 (National Institute of Standards and Technology) mass spectral database (MSD) and Wiley 9th edition MSD (John Wiley and Sons, Inc.), with spectra found in published literature and the Golm Metabolome database. The identification was further confirmed by comparing the retention times, linear retention indices, and mass spectra with those of authentic reference standards treated identically to samples. 
Quantification of different citrus leaf metabolites was based on the peak areas obtained from a series of reference standards $(0$, $5,10,25$, and $50 \mathrm{ppm})$, derivatized and injected under the same conditions as samples. Calibration curves were constructed from the linear regressions obtained by plotting the concentration versus peak area for each standard.

\section{In silico analysis of GABP.}

The bioinformatics and in silico analyses were carried out using the available data of the $C$. sinensis $\mathrm{cv}$. Valencia v2.0 genome from chromosome level HZAU assembly (Xu et al. 2013) in the Citrus Genome database and GenBank, the National Center for Biotechnology Information (NCBI) website. Briefly, the protein sequence of bidirectional amino acid transporter 1 (AtBAT1) of Arabidopsis thaliana (NP_565254.1, also known as $A t G A B P$ ) was matched with eight genes of C. sinensis, using the protein-protein BLAST (BLASTP 2.8.0+) (Altschul et al. 1997, 2005), based on recent available data in NCBI GenBank.

These protein sequences, in addition to AtBAT1 from $A$. thaliana, were used to generate the multiple sequence alignment by ClustalW (Larkin et al. 2007) and BoxShade version 3.21 was used to visualize conserved regions in the alignment. The evolutionary history of the all-matched genes was inferred, using unrooted phylogenetic tree analysis using the neighbor-joining method (Saitou and Nei 1987), with a 1,000-bootstrap test (Felsenstein 1985), and the evolutionary distances were computed using the Poisson correction method (Zuckerkandl and Pauling 1965). Evolutionary analyses were conducted in MEGA7 software (Kumar et al. 2016). Additionally, the protein functional analysis was obtained using InterPro Scan software to look for the conserved domains.

The SWISS-MODEL server (Biasini et al. 2014), I-TASSER server (Roy et al. 2012; Yang and Zhang 2015), and the protein homology/analogy recognition engine (Phyre2 web portalversion 2.0) (Kelley et al. 2015) were used for protein structure homology modeling, generating a 3D structure, and a model for the molecular surface of the predicted amino acid permease BAT1-like isoform X1 of $C$. sinensis (XP_006468761.1; $C s A A P-B A T 1-X 1$; described as $C s G A B P$ to indicate its GABP activity). The Chimera package (version 1.12) was used for interactive visualization of the predicted macromolecule (PDB format). In addition, the prediction of RNA secondary structure for $C s G A B P$ was performed from the DNA sequence using RNAfold webserver (Lorenz et al. 2011).

\section{Gene expression analysis using quantitative real-time PCR (qPCR).}

The total RNA was extracted from the same biological samples, using TriZol reagent (Ambion, Life Technologies). The quantity and quality of isolated RNA were assessed using the NanoDrop 2000 spectrophotometer (Thermo Scientific). SuperScript first-strand synthesis system (Invitrogen) with random hexamer primers was used to synthesize cDNA, as described by the manufacturer instructions. SYBR green PCR master mix (Applied Biosystems) was used to perform the qPCR on an ABI 7500 fast-time PCR system (Applied Biosystems). Samples were analyzed in triplicate for each biological replicate for each treatment (three biological replicates, two technical replicates each, triplicate analysis, $n=$ 30). Primers for 32 various genes involved in GABA shunt cycle and other PAs pathways, in addition to $C s G A B P$, were used to measure gene expression. The relative expression of the consensus sequence among PCR products was done according to the $2^{-\Delta \Delta \mathrm{Ct}}$ method (Livak and Schmittgen 2001). Normalization of gene expression was performed using four endogenous genes (reference genes) including; elongation factor 1-alpha (EF1), F-box/kelch-repeat protein (F-box), glyceraldehyde-3-phosphate dehydrogenase GAPC1, cytosolic (GAPC1, also known as GAPDH), and SAND family protein (SAND) (Mafra et al. 2012; Wei et al. 2014).

\section{Statistical analysis.}

All experiments were designed in completely randomized design. In all experiments, ten biological and two technical replicates per treatment were analyzed $(n=10)$. For statistical analysis, we analyzed only 10 biological replicates. Each biological replicate is a mean of two technical replicates, because our method showed a high reproducibility and the values of each pair of technical replicates were very close to each other. The technical replicates themselves were not used in the statistical analysis, to avoid the possibility of pseudoreplication. Data were normally distributed. Two-way HCA was performed with the standardized and nonstandardized means of the matrices for the four studied treatments. Distance and linkage were done using the Bray-Curtis similarity measure method (Michie 1982), with $95 \%$ confidence between groups from the discriminant function analysis, to construct the similarity dendrograms. Multivariate compound similarities were presented as a heat map, combined with two-way HCA as described above. In addition, PCA was performed using normalized data of individual metabolites and the associated loading plots were generated using singular value decomposition. All data were statistically analyzed according to the ANOVA. Post hoc pairwise comparisons between the four studied treatments were performed with the Tukey-Kramer HSD test, and statistical significance was established at $P \leq 0.05$. The transcript levels of various genes involved in the GABA shunt cycle and PAs pathway were presented as a heat map combined with two-way HCA. HCA was performed using standardized means of the matrices for all studied treatments. Distance and linkage were done as described above.

\section{ACKNOWLEDGMENTS}

The authors acknowledge S. Jones and F. Hijaz for their technical assistance.

\section{LITERATURE CITED}

Altschul, S. F., Madden, T. L., Schäffer, A. A., Zhang, J., Zhang, Z., Miller, W., and Lipman, D. J. 1997. Gapped BLAST and PSI-BLAST: A new generation of protein database search programs. Nucleic Acids Res. 25: 3389-3402.

Altschul, S. F., Wootton, J. C., Gertz, E. M., Agarwala, R., Morgulis, A., Schäffer, A. A., and Yu, Y.-K. 2005. Protein database searches using compositionally adjusted substitution matrices. FEBS J. 272:5101-5109.

Araújo, W. L., Nunes-Nesi, A., Nikoloski, Z., Sweetlove, L. J., and Fernie, A. R. 2012. Metabolic control and regulation of the tricarboxylic acid cycle in photosynthetic and heterotrophic plant tissues. Plant Cell Environ. 35:1-21.

Arbo, M. D., Larentis, E. R., Linck, V. M., Aboy, A. L., Pimentel, A. L., Henriques, A. T., Dallegrave, E., Garcia, S. C., Leal, M. B., and Limberger, R. P. 2008. Concentrations of $p$-synephrine in fruits and leaves of Citrus species (Rutaceae) and the acute toxicity testing of Citrus aurantium extract and p-synephrine. Food Chem. Toxicol. 46: 2770-2775.

Bartley, G. E., Breksa, A. P., 3rd, and Ishida, B. K. 2010. PCR amplification and cloning of tyrosine decarboxylase involved in synephrine biosynthesis in Citrus. N. Biotechnol. 27:308-316.

Bell, E. A. 2003. Nonprotein amino acids of plants: Significance in medicine, nutrition, and agriculture. J. Agric. Food Chem. 51: 2854-2865

Biasini, M., Bienert, S., Waterhouse, A., Arnold, K., Studer, G., Schmidt, T., Kiefer, F., Gallo Cassarino, T., Bertoni, M., Bordoli, L., and Schwede, T. 2014. SWISS-MODEL: Modelling protein tertiary and quaternary structure using evolutionary information. Nucleic Acids Res. 42: W252-W258. 
Blank, L. M., and Sauer, U. 2004. TCA cycle activity in Saccharomyces cerevisiae is a function of the environmentally determined specific growth and glucose uptake rates. Microbiology 150:1085-1093.

Bolton, M. D. 2009. Primary metabolism and plant defense-Fuel for the fire. Mol. Plant-Microbe Interact. 22:487-497.

Bouché, N., Fait, A., Bouchez, D., Møller, S. G., and Fromm, H. 2003. Mitochondrial succinic-semialdehyde dehydrogenase of the $\gamma$-aminobutyrate shunt is required to restrict levels of reactive oxygen intermediates in plants. Proc. Natl. Acad. Sci. U.S.A. 100:6843-6848.

Bouché, N., and Fromm, H. 2004. GABA in plants: Just a metabolite? Trends Plant Sci. 9:110-115.

Bové, J. M. 2006. Huanglongbing: A destructive, newly-emerging, centuryold disease of citrus. J. Plant Pathol. 88:7-37.

Bown, A. W., and Shelp, B. J. 1997. The metabolism and functions of $\gamma$-aminobutyric acid. Plant Physiol. 115:1-5.

Cavalcanti, J. H. F., Esteves-Ferreira, A. A., Quinhones, C. G. S., PereiraLima, I. A., Nunes-Nesi, A., Fernie, A. R., and Araújo, W. L. 2014. Evolution and functional implications of the tricarboxylic acid cycle as revealed by phylogenetic analysis. Genome Biol. Evol. 6:2830-2848.

Cercós, M., Soler, G., Iglesias, D. J., Gadea, J., Forment, J., and Talón, M. 2006. Global analysis of gene expression during development and ripening of citrus fruit flesh. A proposed mechanism for citric acid utilization. Plant Mol. Biol. 62:513-527.

Cevallos-Cevallos, J. M., Futch, D. B., Shilts, T., Folimonova, S. Y., and Reyes-De-Corcuera, J. I. 2012. GC-MS metabolomic differentiation of selected citrus varieties with different sensitivity to citrus Huanglongbing. Plant Physiol. Biochem. 53:69-76.

Cevallos-Cevallos, J. M., García-Torres, R., Etxeberria, E., and Reyes-DeCorcuera, J. I. 2011. GC-MS analysis of headspace and liquid extracts for metabolomic differentiation of citrus Huanglongbing and zinc deficiency in leaves of 'Valencia' sweet orange from commercial groves. Phytochem. Anal. 22:236-246.

Chevrot, R., Rosen, R., Haudecoeur, E., Cirou, A., Shelp, B. J., Ron, E., and Faure, D. 2006. GABA controls the level of quorum-sensing signal in Agrobacterium tumefaciens. Proc. Natl. Acad. Sci. U.S.A. 103:7460-7464.

Cohen, S. S. 1998. A guide to the polyamines. Oxford University Press, Oxford.

Cruz-Munoz, M., Petrone, J. R., Cohn, A. R., Munoz-Beristain, A., Killiny, N., Drew, J. C., and Triplett, E. W. 2018. Development of chemically defined media reveals citrate as preferred carbon source for Liberibacter growth. Front. Microbiol. 9:668.

da Graça, J. V. 1991. Citrus greening disease. Annu. Rev. Phytopathol. 29: 109-136.

Dragull, K., Breksa, A. P., 3rd, and Cain, B. 2008. Synephrine content of juice from Satsuma mandarins (Citrus unshiu Marcovitch). J. Agric. Food Chem. 56:8874-8878.

Fait, A., Fromm, H., Walter, D., Galili, G., and Fernie, A. R. 2008. Highway or byway: The metabolic role of the GABA shunt in plants. Trends Plant Sci. 13:14-19.

Felsenstein, J. 1985. Confidence limits on phylogenies: An approach using the bootstrap. Evolution 39:783-791.

Folimonova, S. Y., Robertson, C. J., Garnsey, S. M., Gowda, S., and Dawson, W. O. 2009. Examination of the responses of different genotypes of citrus to huanglongbing (citrus greening) under different conditions. Phytopathology 99:1346-1354.

Garnier, M., Jagoueix-Eveillard, S., Cronje, P. R., Le Roux, H. F., and Bové, J. M. 2000. Genomic characterization of a liberibacter present in an ornamental rutaceous tree, Calodendrum capense, in the Western Cape Province of South Africa. Proposal of 'Candidatus Liberibacter africanus subsp. capensis'. Int. J. Syst. Evol. Microbiol. 50:2119-2125.

Gottwald, T. R. 2010. Current epidemiological understanding of citrus Huanglongbing. Annu. Rev. Phytopathol. 48:119-139.

Haines, R. J., Pendleton, L. C., and Eichler, D. C. 2011. Argininosuccinate synthase: At the center of arginine metabolism. Int. J. Biochem. Mol. Biol. 2:8-23.

Halbert, S. E., and Manjunath, K. L. 2004. Asian citrus psyllids (Sternorrhyncha: Psyllidae) and greening disease of citrus: A literature review and assessment of risk in florida. Fla. Entomol. 87:330-353.

Hijaz, F., El-Shesheny, I., and Killiny, N. 2013. Herbivory by the insect diaphorina citri induces greater change in citrus plant volatile profile than does infection by the bacterium, Candidatus Liberibacter asiaticus. Plant Signal. Behav. 8:4161, 25677.

Hijaz, F., and Killiny, N. 2014. Collection and chemical composition of phloem sap from Citrus sinensis L. Osbeck (sweet orange). PLoS One 9: e101830.

Hijaz, F., Nehela, Y., and Killiny, N. 2016. Possible role of plant volatiles in tolerance against huanglongbing in citrus. Plant Signal. Behav. 11: e1138193.
Hussain, S. B., Shi, C. Y., Guo, L.-X., Kamran, H. M., Sadka, A., and Liu, Y.-Z. 2017. Recent advances in the regulation of citric acid metabolism in citrus fruit. CRC. Crit. Rev. Plant Sci. 36:241-256.

Jagoueix, S., Bove, J. M., and Garnier, M. 1994. The phloem-limited bacterium of greening disease of citrus is a member of the alpha subdivision of the proteobacteria. Int. J. Syst. Bacteriol. 44:379-386.

Kangasjärvi, S., Neukermans, J., Li, S., Aro, E.-M., and Noctor, G. 2012. Photosynthesis, photorespiration, and light signalling in defence responses. J. Exp. Bot. 63:1619-1636.

Kaur-Sawhney, R., Tiburcio, A. F., Altabella, T., and Galston, A. W. 2003. Polyamines in plants: An overview. J. Cell Mol. Biol. 2:1-12.

Kelley, L. A., Mezulis, S., Yates, C. M., Wass, M. N., and Sternberg, M. J. E. 2015. The Phyre2 web portal for protein modeling, prediction and analysis. Nat. Protoc. 10:845-858.

Killiny, N. 2017. Metabolite signature of the phloem sap of fourteen citrus varieties with different degrees of tolerance to Candidatus Liberibacter asiaticus. Physiol. Mol. Plant Pathol. 97:20-29.

Killiny, N., and Hijaz, F. 2016. Amino acids implicated in plant defense are higher in Candidatus Liberibacter asiaticus-tolerant citrus varieties. Plant Signal. Behav. 11:e1171449.

Killiny, N., Hijaz, F., Ebert, T. A., and Rogers, M. E. 2017a. A plant bacterial pathogen manipulates its insect vector's energy metabolism. Appl. Environ. Microbiol. 83:e03005-e03016.

Killiny, N., and Jones, S. E. 2018. Metabolic alterations in the nymphal instars of Diaphorina citri induced by Candidatus Liberibacter asiaticus, the putative pathogen of huanglongbing. PLoS One 13:e0191871.

Killiny, N., Jones, S. E., Nehela, Y., Hijaz, F., Dutt, M., Gmitter, F. G., and Grosser, J. W. 2018a. All roads lead to Rome: Towards understanding different avenues of tolerance to Huanglongbing in citrus cultivars. Plant Physiol. Biochem. 129:1-10.

Killiny, N., and Nehela, Y. 2017a. Metabolomic response to Huanglongbing: Role of carboxylic compounds in Citrus sinensis response to " Candidatus Liberibacter asiaticus' and its vector, Diaphorina citri. Mol. Plant-Microbe Interact. 30:666-678.

Killiny, N., and Nehela, Y. 2017b. One target, two mechanisms: The impact of "Candidatus Liberibacter asiaticus" and its vector, Diaphorina citri, on citrus leaf pigments. Mol. Plant-Microbe Interact. 30:543-556.

Killiny, N., Nehela, Y., Hijaz, F., and Vincent, C. I. 2018b. A plant pathogenic bacterium exploits the tricarboxylic acid cycle metabolic pathway of its insect vector. Virulence 9:99-109.

Killiny, N., Valim, M. F., Jones, S. E., Omar, A. A., Hijaz, F., Gmitter, F. G., Jr., and Grosser, J. W. 2017b. Metabolically speaking: Possible reasons behind the tolerance of 'Sugar Belle' mandarin hybrid to huanglongbing. Plant Physiol. Biochem. 116:36-47.

Kumar, S., Stecher, G., and Tamura, K. 2016. MEGA7: Molecular evolutionary genetics analysis version 7.0 for bigger datasets. Mol. Biol. Evol. 33:1870-1874.

Larkin, M. A., Blackshields, G., Brown, N. P., Chenna, R., McGettigan, P. A., McWilliam, H., Valentin, F., Wallace, I. M., Wilm, A., Lopez, R., Thompson, J. D., Gibson, T. J., and Higgins, D. G. 2007. Clustal W and Clustal X version 2.0. Bioinformatics 23:2947-2948.

Liu, X., Hu, X.-M., Jin, L.-F., Shi, C.-Y., Liu, Y.-Z., and Peng, S.-A. 2014 Identification and transcript analysis of two glutamate decarboxylase genes, $C s G A D 1$ and $C s G A D 2$, reveal the strong relationship between CsGAD1 and citrate utilization in citrus fruit. Mol. Biol. Rep. 41: 6253-6262.

Livak, K. J., and Schmittgen, T. D. 2001. Analysis of relative gene expression data using real-time quantitative PCR and the $2_{\mathrm{T}}^{\left(-\Delta \Delta^{\mathrm{C}}()\right)}$ Method. Methods 25:402-408.

Lorenz, R., Bernhart, S. H., Höner Zu Siederdissen, C., Tafer, H., Flamm, C., Stadler, P. F., and Hofacker, I. L. 2011. ViennaRNA Package 2.0. Algorithms Mol. Biol. 6:26.

Lu, Z., and Killiny, N. 2017. Huanglongbing pathogen Candidatus Liberibacter asiaticus exploits the energy metabolism and host defence responses of its vector Diaphorina citri. Physiol. Entomol. 42:319-335.

Mafra, V., Kubo, K. S., Alves-Ferreira, M., Ribeiro-Alves, M., Stuart, R. M., Boava, L. P., Rodrigues, C. M., and Machado, M. A. 2012 Reference genes for accurate transcript normalization in citrus genotypes under different experimental conditions. PLoS One 7:e31263.

Malik, N. S., Perez, J. L., Kunta, M., Patt, J. M., and Mangan, R. L. 2014 Changes in free amino acids and polyamine levels in Satsuma leaves in response to Asian citrus psyllid infestation and water stress. Insect Sci. 21:707-716.

Michaeli, S., Fait, A., Lagor, K., Nunes-Nesi, A., Grillich, N., Yellin, A., Bar, D., Khan, M., Fernie, A. R., Turano, F. J., and Fromm, H. 2011. A mitochondrial GABA permease connects the GABA shunt and the TCA cycle, and is essential for normal carbon metabolism. Plant J. 67: 485-498. 
Michie, M. G. 1982. Use of the Bray-Curtis similarity measure in cluster analysis of foraminiferal data. J. Int. Assoc. Math. Geol. 14:661-667.

Moschou, P. N., Wu, J., Cona, A., Tavladoraki, P., Angelini, R., and Roubelakis-Angelakis, K. A. 2012. The polyamines and their catabolic products are significant players in the turnover of nitrogenous molecules in plants. J. Exp. Bot. 63:5003-5015.

Nehela, Y., Hijaz, F., Elzaawely, A. A., El-Zahaby, H. M., and Killiny, N. 2016. Phytohormone profiling of the sweet orange (Citrus sinensis (L.) Osbeck) leaves and roots using GC-MS-based method. J. Plant Physiol. 199:12-17.

Nehela, Y., Hijaz, F., Elzaawely, A. A., El-Zahaby, H. M., and Killiny, N. 2018. Citrus phytohormonal response to Candidatus Liberibacter asiaticus and its vector Diaphorina citri. Physiol. Mol. Plant Pathol. 102:24-35.

Palanivelu, R., Brass, L., Edlund, A. F., and Preuss, D. 2003. Pollen tube growth and guidance is regulated by POP2, an Arabidopsis gene that controls GABA levels. Cell 114:47-59.

Parker, D., Beckmann, M., Zubair, H., Enot, D. P., Caracuel-Rios, Z., Overy, D. P., Snowdon, S., Talbot, N. J., and Draper, J. 2009. Metabolomic analysis reveals a common pattern of metabolic reprogramming during invasion of three host plant species by Magnaporthe grisea. Plant J. 59:723-737.

Pitino, M., Armstrong, C. M., and Duan, Y. 2017. Molecular mechanisms behind the accumulation of ATP and $\mathrm{H}_{2} \mathrm{O}_{2}$ in citrus plants in response to 'Candidatus Liberibacter asiaticus' infection. Hortic. Res. 4:17040.

Roberts, M. R. 2007. Does GABA act as a signal in plants? Hints from molecular studies. Plant Signal. Behav. 2:408-409.

Rocha, M., Licausi, F., Araújo, W. L., Nunes-Nesi, A., Sodek, L., Fernie, A. R., and van Dongen, J. T. 2010. Glycolysis and the tricarboxylic acid cycle are linked by alanine aminotransferase during hypoxia induced by waterlogging of Lotus japonicus. Plant Physiol. 152:1501-1513.

Rosales, R., and Burns, J. K. 2011. Phytohormone changes and carbohydrate status in sweet orange fruit from Huanglongbing-infected trees. J. Plant Growth Regul. 30:312-321.

Roy, A., Yang, J., and Zhang, Y. 2012. COFACTOR: An accurate comparative algorithm for structure-based protein function annotation. Nucleic Acids Res. 40:W471-W477.

Saitou, N., and Nei, M. 1987. The neighbor-joining method: A new method for reconstructing phylogenetic trees. Mol. Biol. Evol. 4:406-425.

Shelp, B. J., Bown, A. W., and McLean, M. D. 1999. Metabolism and functions of gamma-aminobutyric acid. Trends Plant Sci. 4:446-452.

Shelp, B. J., Bown, A. W., and Zarei, A. 2017. 4-Aminobutyrate (GABA): A metabolite and signal with practical significance. Botany 95:1015-1032.

Sheng, L., Shen, D., Luo, Y., Sun, X., Wang, J., Luo, T., Zeng, Y., Xu, J., Deng, X., and Cheng, Y. 2017. Exogenous $\gamma$-aminobutyric acid treatment affects citrate and amino acid accumulation to improve fruit quality and storage performance of postharvest citrus fruit. Food Chem. 216: 138-145.

Slisz, A. M., Breksa, A. P., 3rd, Mishchuk, D. O., McCollum, G., and Slupsky, C. M. 2012. Metabolomic analysis of citrus infection by 'Candidatus Liberibacter' reveals insight into pathogenicity. J. Proteome Res. 11:4223-4230.

Sweetlove, L. J., Beard, K. F. M., Nunes-Nesi, A., Fernie, A. R., and Ratcliffe, R. G. 2010. Not just a circle: Flux modes in the plant TCA cycle. Trends Plant Sci. 15:462-470.

Tatineni, S., Sagaram, U. S., Gowda, S., Robertson, C. J., Dawson, W. O., Iwanami, T., and Wang, N. 2008. In planta distribution of 'Candidatus Liberibacter asiaticus' as revealed by polymerase chain reaction (PCR) and real-time PCR. Phytopathology 98:592-599.

do Carmo Teixeira, D., Luc Danet, J., Eveillard, S., Cristina Martins, E., de Jesus Junior, W. C., Takao Yamamoto, P., Aparecido Lopes, S., Beozzo Bassanezi, R., Juliano Ayres, A., Saillard, C., and Bové, J. M. 2005. Citrus Huanglongbing in São Paulo State, Brazil: PCR detection of the
'Candidatus' Liberibacter species associated with the disease. Mol. Cell. Probes 19:173-179.

Tiburcio, A. F., Altabella, T., Borrell, A., and Masgrau, C. 1997. Polyamine metabolism and its regulation. Physiol. Plant. 100:664-674.

Voll, L. M., Horst, R. J., Voitsik, A. M., Zajic, D., Samans, B., PonsKühnemann, J., Doehlemann, G., Münch, S., Wahl, R., Molitor, A. Hofmann, J., Schmiedl, A., Waller, F., Deising, H. B., Kahmann, R., Kämper, J., Kogel, K.-H., and Sonnewald, U. 2011. Common motifs in the response of cereal primary metabolism to fungal pathogens are not based on similar transcriptional reprogramming. Front. Plant Sci. 2:39.

Walters, D. 2003a. Resistance to plant pathogens: Possible roles for free polyamines and polyamine catabolism. New Phytol. 159:109-115.

Walters, D. R. 2000. Polyamines in plant-microbe interactions. Physiol. Mol. Plant Pathol. 57:137-146.

Walters, D. R. 2003b. Polyamines and plant disease. Phytochemistry 64: 97-107.

Wei, X., Chen, C., Yu, Q., Gady, A., Yu, Y., Liang, G., and Gmitter, F. G. 2014. Novel expression patterns of carotenoid pathway-related genes in citrus leaves and maturing fruits. Tree Genet. Genomes 10:439-448.

Wheaton, T. A., and Stewart, I. 1969. Biosynthesis of synephrine in citrus. Phytochemistry 8:85-92.

Wheaton, T. A., and Stewart, I. 1970. The distribution of tyramine, Nmethyltyramine, hordenine, octopamine, and synephrine in higher plants. Lloydia 33:244-254.

Xiong, W., Brune, D., and Vermaas, W. F. J. 2014. The $\gamma$-aminobutyric acid shunt contributes to closing the tricarboxylic acid cycle in Synechocystis sp. PCC 6803. Mol. Microbiol. 93:786-796.

Xu, Q., Chen, L.-L., Ruan, X., Chen, D., Zhu, A., Chen, C., Bertrand, D., Jiao, W.-B., Hao, B.-H., Lyon, M. P., Chen, J., Gao, S., Xing, F., Lan, H., Chang, J.-W., Ge, X., Lei, Y., Hu, Q., Miao, Y., Wang, L., Xiao, S., Biswas, M. K., Zeng, W., Guo, F., Cao, H., Yang, X., Xu, X.-W., Cheng, Y.-J., Xu, J., Liu, J.-H., Luo, O. J., Tang, Z., Guo, W.-W., Kuang, H., Zhang, H.-Y., Roose, M. L., Nagarajan, N., Deng, X.-X., and Ruan, Y. 2013. The draft genome of sweet orange (Citrus sinensis). Nat. Genet. 45:59-66.

Yang, J., and Zhang, Y. 2015. I-TASSER server: New development for protein structure and function predictions. Nucleic Acids Res. 43: W174-W181.

Zuckerkandl, E., and Pauling, L. 1965. Evolutionary divergence and convergence in proteins. Pages 97-166 in: Evolving Genes and Proteins. Bryson, V., and Vogel, H. J., eds. Academic Press, New York.

\section{AUTHOR-RECOMMENDED INTERNET RESOURCES}

BoxShade version 3.21: https://embnet.vital-it.ch/software/BOX_form.html

Chimera package version 1.12:

https://www.cgl.ucsf.edu/chimera/download.html

Citrus Genome database:

https://www.citrusgenomedb.org/organism/Citrus/sinensis

ClustalW: http://www.genome.jp/tools-bin/clustalw

Golm Metabolome database: http://gmd.mpimp-golm.mpg.de

InterPro Scan software: https://www.ebi.ac.uk/interpro

I-TASSER server: https://zhanglab.ccmb.med.umich.edu/I-TASSER

National Center for Biotechnology Information GenBank website: http://www.ncbi.nlm.nih.gov/gene

Phyre2 web portal version 2.0:

http://www.sbg.bio.ic.ac.uk/ phyre2/html/page.cgi?id=index

The RCSB Protein Data Bank: https://www.rcsb.org

RNAfold webserver:

http://rna.tbi.univie.ac.at//cgi-bin/RNAWebSuite/RNAfold.cgi

SWISS-MODEL server: https://swissmodel.expasy.org 